Notes ouest-africaines

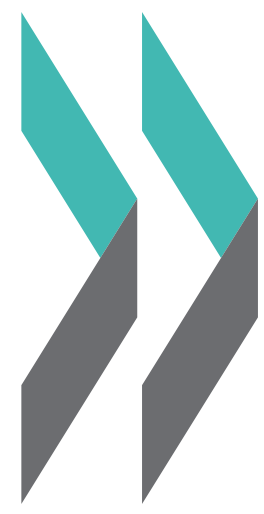

\title{
Intégrer l'analyse sexospécifique dans les systèmes d'alerte précoce pour la sécurité alimentaire et nutritionnelle en Afrique de l'Ouest
}

AVRIL 2019 No 24 


\section{INTÉGRER L'ANALYSE SEXOSPÉCIFIQUE DANS LES SYSTĖMES D'ALERTE PRÉCOCE POUR LA SÉCURITÉ ALIMENTAIRE ET NUTRITIONNELLE EN AFRIQUE DE L'OUEST}

Cette note a été rédigée par

ANALEE PEPPER

BETTINE PEPPER ADVISING 


\section{NOTES OUEST-AFRICAINES}

Les Notes ouest-africaines analysent les dynamiques socio-économiques, politiques et sécuritaires que traverse l'Afrique dans une perspective régionale et multidisciplinaire. Elles cherchent à stimuler la discussion, rassembler les informations et mieux anticiper les transformations en cours pour les politiques à venir. Elles visent à partager des études avec une large audience d'experts, de praticiens du développement, de décisionnaires et de lecteurs avertis. Les Notes sont disponibles en anglais et/ou en français ; les résumés dans les deux langues. Initiées par le Club du Sahel et de l'Afrique de l'Ouest (CSAO) pour éclairer les enjeux ouest-africains, ces analyses sont préparées par son Secrétariat, ses membres et partenaires, les autres départements de l'OCDE, des organisations internationales et autres experts et chercheurs.

Merci de citer cet ouvrage comme suit:

Pepper, A. (2019), « Intégrer l'analyse sexospécifique dans les systèmes d'alerte précoce pour la sécurité alimentaire et nutritionnelle en Afrique de l’Ouest ), Notes ouest-africaines, № 24, Éditions OCDE, Paris.

Contact auteur : apepper@bettinepepper.com

https://doi.org/10.1787/d02dfd6b-fr

ISSN 2414-2026

Ce document est publié sous la responsabilité du Secrétaire général de l'OCDE. Les opinions et les interprétations qui y sont exprimées et les arguments qui y sont employés ne reflètent pas nécessairement les vues officielles des gouvernements des pays membres de l'OCDE.

Ce document et toute carte qu'il peut comprendre ne préjugent en rien du statut de tout territoire, de la souveraineté s'exerçant sur ce dernier, du tracé des frontières et limites internationales, et du nom de tout territoire, ville ou région.

Autorisé pour publication par Laurent Bossard, Directeur, Secrétariat du Club du Sahel et de l'Afrique de l'Ouest (CSAO/OCDE).

Vous êtes autorisés à copier, télécharger ou imprimer du contenu OCDE pour votre utilisation personnelle. Vous pouvez inclure des extraits des publications, des bases de données et produits multimédia de l'OCDE dans vos documents, présentations, blogs, sites Internet et matériel d'enseignement, sous réserve de faire mention de la source OCDE et du copyright. Les demandes pour usage commercial ou de traduction devront être adressées à rights@oecd.org. 


\section{Résumé}

L'intégration de la problématique femmes-hommes dans les systèmes d'alerte précoce est essentielle afin de prévenir et gérer les crises de façon plus équitable. Cette Note examine la prise en compte dans les systèmes d'alerte précoce pour la sécurité alimentaire et nutritionnelle (SAP-SAN) au Sahel et en Afrique de l'Ouest du genre. Elle identifie les lacunes aux niveaux national et régional. Il en ressort que les partenaires et parties prenantes ont renforcé la capacité d'analyse sexospécifique de ces systèmes. Mais ces avancées n'ont pas abouti à une refonte uniforme des mécanismes conjoints auxquels font appel les SAP-SAN afin de les rendre plus sensibles aux disparités qui existent entre les sexes. Des efforts plus concertés s'imposent pour déterminer les aspects de la problématique femmes-hommes à intégrer dans les SAP-SAN et en assurer le suivi pour des systèmes de prévention et de gestion de crise plus équitables. Ce document présente des pistes pour appuyer ces efforts.

Mots-clés : systèmes d'alerte précoce, sécurité alimentaire, analyse sexospécifique, prise en compte de la problématique femmes-hommes, données ventilées par sexe et par âge.

Classification JEL : H120, J160, R580, Q180

\section{À propos de l'auteur}

Analee Pepper est une experte senior sur les questions de genre dans les contextes de l'aide humanitaire et du développement international. Elle a effectué de nombreuses missions de conseil pour des organisations internationales au sujet de l'intégration du genre dans les projets de développement, afin d'accroître l'impact de ces projets. Elle a également publié une série d'articles et des guides pratiques sur les liens entre les problématiques de genre et la sécurité alimentaire, l'emploi, les conflits et la sécurité. En outre, elle a travaillé sur le renforcement des capacités pour l'analyse de ces problématiques en Afrique de l'Ouest. Ses activités de conseil l'ont amené à collaborer avec ONU Femmes, le PAM, UNICEF, l'OMS et MSF, entre autres organisations. Elle possède un Bachelier en Développement International et Études Féminines de l'Université de Californie à Los Angeles (UCLA) et un Master en Études du Développement de l'Institut de Hautes Études Internationales et du Développement (IHEID Genève). Son mémoire de master s'est concentré sur la prise en compte de l'analyse des dynamiques de genre dans les systèmes d'alerte précoce pour les conflits. 


\section{Le Club du Sahel et de l'Afrique de l'Ouest}

Le Club du Sahel et de l'Afrique de l'Ouest (CSAO) est une plateforme internationale indépendante. Le secrétariat est hébergé au sein de l'Organisation de coopération et de développement économiques (OCDE). Sa mission est de promouvoir des politiques régionales à même d'améliorer le bien-être économique et social des populations ouest-africaines. Ses objectifs sont de : i) Améliorer la gouvernance régionale de la sécurité alimentaire et nutritionnelle, ii) Comprendre les transformations en cours dans la région et leurs implications en matière de politiques publiques par des analyses régionales, spatiales et prospectives. Ses Membres sont l'Autriche, la Belgique, le Canada, la Commission de la CEDEAO, le CILSS, la Commission de l'UEMOA, l'Union européenne, les États-Unis, la France, le Luxembourg, les Pays-Bas et la Suisse. Le CSAO a conclu des protocoles d'accord avec l'Agence du NEPAD et l'Université de Floride (Groupe de recherche sur le Sahel).

En savoir plus : www.oecd.org/csao 


\section{Table des matières}

LISTE DES SIGLES ET ACRONYMES

SYNTHÈSE

INTRODUCTION

Les systèmes d'alerte précoce dans le cadre de la surveillance de la sécurité alimentaire et nutritionnelle

Les aspects sexospécifiques de la sécurité alimentaire et nutritionnelle

Les aspects sexospécifiques des systèmes d'alerte précoce pour la sécurité alimentaire et nutritionnelle.

APPROCHES SUIVIES PAR LES PARTIES PRENANTES DU PREGEC POUR RENFORCER LA PRISE EN COMPTE DE LA PROBLÉMATIQUE FEMMESHOMMES DANS LES SAP-SAN

Politiques en faveur de l'égalité femmes-hommes et ancrage institu-

tionnel

Cadres d'analyse, outils et formation. 23

Données ventilées par sexe et par âge (DVSA) et indicateurs sexospécifiques

LACUNES ET LIMITES AUXQUELLES SE HEURTE L'ANALYSE SEXOSPÉCIFIQUE DANS LE CADRE DES SAP-SAN

CONCLUSION. 36

Notes

RÉFÉRENCES. 38

Annex I : Problématique femmes-hommes et marchés en Afrique de l'Ouest : liste des sources de données secondaires et description succincte août 2016 (PAM).

Annex II : Liste des organismes consultés. 


\section{Liste des sigles et acronymes}

ACF Action contre la faim

CEDEAO Communauté économique des États de l'Afrique de l'Ouest

CILSS Comité permanent inter-États de lutte contre la sécheresse dans le Sahel

CPI Comité permanent interorganisations (CPI)

CSAO Club du Sahel et de l'Afrique de l'Ouest

DVSA Données ventilées par sexe et par âge

EMPRES* Système de prévention et de réponse précoce contre les ravageurs et les maladies transfrontières des animaux et des plantes

FAO* Organisation des Nations Unies pour l'alimentation et l'agriculture

FEWS NET* Réseau des systèmes d'alerte précoce contre la famine

IFPRI* Institut international de recherche sur les politiques alimentaires

IPC* Cadre intégré de classification de la sécurité alimentaire

OCDE Organisation de coopération et de développement économiques

PAM Programme alimentaire mondial

PREGEC Dispositif de prévention et de gestion des crises alimentaires

REOWA* Bureau régional de la FAO pour la résilience, les urgences et la réhabilitation en Afrique de l'Ouest/Sahel

RPCA Réseau de prévention des crises alimentaires 
SAP Système d'alerte précoce

SAP-SAN Système(s) d'alerte précoce pour la sécurité alimentaire et nutritionnelle

SMIAR Système mondial d'information et d'alerte rapide sur l'alimentation et l'agriculture

TIC Technologies de l'information et de la communication

UEMOA Union économique et monétaire ouest-africaine

UNICEF* Fonds des Nations Unies pour l'enfance

USAID* Agence des États-Unis pour le développement international

VAM* Unité d'analyse et de cartographie de la vulnérabilité (du PAM)

WEAI* Indice d'autonomisation des femmes dans l'agriculture

WEMI* Indice d'autonomisation des femmes dans les marchés (PAM)

*Sigle dans la langue originale 


\section{Synthèse}

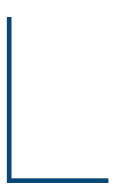

es systèmes d'alerte précoce (SAP) jouent un rôle essentiel pour la prévention et la gestion des crises alimentaires. Malheureusement, ces systèmes ne prennent que rarement en compte le fait que l'insécurité alimentaire et nutritionnelle n'affecte pas les femmes et les hommes de la même manière. II faut tenir compte des dimensions "sexospécifiques » de l'insécurité alimentaire et nutritionnelle dans les SAP pour que ces systèmes mènent à des mesures de prévention et de gestion des crises équitables et efficaces. La collecte de données sexospécifiques pour abonder les SAP joue à cet égard un rôle crucial.

La présente Note examine la mesure dans laquelle les SAP au Sahel et en Afrique de l'Ouest sont sensibles à la réalité des disparités entre les femmes et les hommes. Elle présente un panel de politiques, d'outils et de cadres qui permettent de réaliser plus facilement des analyses sur les liens entre le genre et la sécurité alimentaire et nutritionnelle. Elle discute également le rôle des données ventilées par sexe et par âge (DVSA), qui constituent le socle de ces analyses.

Au Sahel et en Afrique de l'Ouest, le Dispositif de prévention et de gestion des crises alimentaires (PREGEC) est fondé sur une approche harmonisée en matière de SAP. Cette approche s'exprime dans un outil, le « Cadre harmonisé s) ${ }^{1}$. Le Cadre harmonisé prévoit des réunions régulières d'experts du PREGEC. Durant ces consultations techniques, les résultats de l'observation de la situation alimentaire et nutritionnelle sont évalués et validés. Cet exercice permet de repérer à l'avance les régions et populations à risque. Bien qu'il existe un ensemble toujours plus vaste d'études et de bases de données montrant la corrélation étroite qui lie les inégalités de genre et l'insécurité alimentaire, le Cadre harmonisé ne contient pas de règles ou de recommandations sur la réalisation d'analyses et la collecte de données sur le genre. Les parties prenantes du Cadre harmonisé ont entrepris de multiples efforts autour du thème du genre (conceptions de cadres, d'outils et de mécanismes de redevabilité), mais les progrès des SAP sur ce terrain demeurent lents. Cette situation ne fait que souligner l'urgence de produire des orientations stratégiques sur la façon dont les efforts des partenaires peuvent être renforcés, coordonnés et mis au service d'une transformation des SAP, pour les rendre perméables aux influences des problématiques femmes-hommes. 


\section{Messages clés}

Les mesures suivantes sont proposées pour répondre aux défis de l'intégration des disparités femmes-hommes dans les systèmes d'alerte précoce pour la sécurité alimentaire et nutritionnelle (SAP-SAN).

1. Renforcer les capacités à produire de l'analyse sur les dimensions sexospécifiques de la sécurité alimentaire et nutritionnelle

Une part croissante des parties prenantes sont conscientes de l'effet des disparités femmes-hommes sur la vulnérabilité face aux crises alimentaires et nutritionnelles. Néanmoins, les consultations avec les représentants des membres du Réseau de prévention des crises alimentaires (RPCA) ont montré qu'il est nécessaire de poursuivre et de renforcer les actions de sensibilisation sur ce thème. Le personnel technique doit posséder une compréhension détaillée et concrète de la manière dont les disparités femmes-hommes affectent la sécurité alimentaire des individus et de leurs foyers. Il doit également être capable d'utiliser et d'adopter un cadre de suivi PREGEC sensible aux impacts du genre.

2. Mutualiser les ressources et intégrer l'analyse sexospécifique dans le PREGEC

La volonté de mettre en commun les ressources et les informations pour renforcer la surveillance exercée par les partenaires et les gouvernements sur la sécurité alimentaire et nutritionnelle dans les communautés vulnérables constitue le fondement du PREGEC. S'il est vrai que certaines parties prenantes ont déjà mis au point des outils perfectionnés d'analyse sexospécifique dans certains domaines, cela n'a pas encore mené à l'adoption d'une approche commune et harmonisée au genre dans le cadre du RPCA. Les parties prenantes devraient mutualiser leurs ressources pour mettre à jour le Cadre harmonisé et généraliser son application. Cette mise à jour devrait inclure des indications concrètes quant à la manière d'effectuer des analyses sexospécifiques et de produire des DVSA.

3. Assurer la cohérence des politiques et la transparence vis-à-vis des parties prenantes

La plupart des parties prenantes du PREGEC se sont dotées de politiques en faveur de l'égalité femmes-hommes, en concordance avec l'Objectif de développement durable 5 (ODD 5), dont le but est de (c parvenir à l'égalité des sexes et autonomiser toutes les femmes et les filles ). Ces politiques devraient indiquer clairement le besoin de doter les SAP d'une capacité d'analyse sexospécifique, et préciser comment de telles analyses peuvent être produites en 
pratique. A l'heure actuelle, les politiques des parties prenantes du PREGEC en matière d'égalité femmes-hommes ne font que rarement référence aux SAP. La cohérence des politiques est fondamentale pour améliorer la prise en compte des disparités entre femmes et hommes dans les mesures de prévention et de gestion des crises alimentaires.

4. Promouvoir une participation paritaire des populations locales à la conception et au fonctionnement des SAP

Associer les femmes, les hommes et les jeunes à la conception et au fonctionnement des SAP est indispensable pour que les systèmes de prévention et de gestion des crises soient vraiment inclusifs et couvrent toute la population. La parité femmes-hommes devrait être la règle pour les organisations qui s'engagent aux côtés des populations locales au sujet des SAP. Sans cela, les personnes exclues du processus seront exposées à des risques plus grands, car leurs préférences et leurs besoins n'auront pas été entendus. Ces personnes risquent aussi d'être moins bien informées des mesures à prendre en cas de crise. L'( inclusivité )) est donc essentielle pour que les SAP puissent atteindre leurs objectifs en termes de prévention et de gestion des crises. 


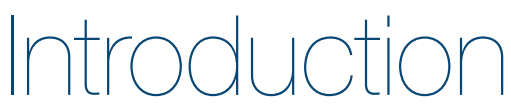

$\square$

a Note examine dans quelle mesure les systèmes d'alerte précoce (SAP) en vigueur au Sahel et en Afrique de l'Ouest prennent en compte les disparités entre les sexes. Elle contribue aux efforts visant à renforcer l'intégration de la problématique femmes-hommes dans le Cadre harmonisé, le dispositif commun à l'appui des systèmes d'alerte précoce pour la sécurité alimentaire et nutritionnelle (SAP-SAN). Elle identifie les approches et les initiatives actuelles en faveur de l'analyse sexospécifique dans le domaine de la sécurité alimentaire et nutritionnelle, les lacunes des SAP-SAN et explore les possibilités d'améliorer la prise en compte, par ces systèmes, des disparités femmes-hommes dans la région. Les informations utilisées proviennent d'une étude documentaire sur les pratiques en vigueur, de recherches secondaires sur l'analyse sexospécifique dans les SAP-SAN et d'entretiens avec des acteurs clés dans la région.

Les recherches existantes montrent une forte corrélation entre les inégalités entre femmes et hommes et l'insécurité alimentaire et nutritionnelle. Les normes en termes de genre et d'inégalités influent sur la réaction des individus lors d'une crise et leur vulnérabilité face à celle-ci. D'après le Guide des genres pour les actions humanitaires du Comité permanent interorganisations (CPI), document de portée mondiale essentiel pour la prise en compte de la problématique femmes-hommes dans les activités humanitaires, les crises ne touchent pas les femmes, les filles, les hommes et les garçons de la même façon pour ce qui est de l'accès à la nourriture, des moyens d'existence et de la nutrition (CPI, 2017). En raison des rôles et des responsabilités dévolus à chaque sexe, les femmes, les filles, les hommes et les garçons sont susceptibles de contribuer différemment aux systèmes de surveillance de la sécurité alimentaire et nutritionnelle, ainsi qu'à la planification et mise en œuvre des mesures prises.

Les femmes et les filles jouent des rôles importants et distincts de ceux des hommes et des garçons dans l'économie alimentaire. Ce constat est particulièrement vrai en Afrique de l'Ouest, où les femmes sont des actrices de premier plan à chaque échelon du système alimentaire, qu'il s'agisse de la production, de la transformation, de la distribution ou de la préparation des aliments (Gnisci, 2016). Les femmes exerçant des activités dans l'économie alimentaire non agricole représentent en effet entre $72 \%$ et $88 \%$ de l'emploi total dans la transformation et la commercialisation des aliments, respectivement (Allen et al. 2018). Les femmes jouent en outre un rôle non négligeable dans le renforcement de la résilience face aux crises alimentaires qui résultent du climat et des conflits (Gnisci, 2016). En dépit de ces réalités, l'intégration de l'analyse sexospécifique dans les dispositifs de surveillance 
de la sécurité alimentaire et nutritionnelle reste lente. C'est notamment le cas des SAP, dont la méthodologie et les sources de données ne facilitent pas la prise en compte des disparités entre femmes et hommes.

Les SAP-SAN sont des outils efficaces de suivi des changements saisonniers et de l'évolution démographique. Les gouvernements, les Nations Unies (ONU) et les organisations non gouvernementales internationales (ONGI) collaborent dans le cadre de plateformes unifiées qui leur permettent de mettre en commun leurs données et de procéder à des expertises inter-institutions. Mais, dans bien des cas, ces SAP prennent insuffisamment en compte la problématique femmes-hommes. Les orientations qui les guident et leurs pratiques témoignent d'une conscience limitée de l'importance des aspects sexospécifiques de la sécurité alimentaire et nutritionnelle, ainsi que des avantages liés à l'adoption de SAP-SAN sensibles aux disparités entre les sexes ; et ce, en dépit d'initiatives innovantes et encourageantes lancées par des acteurs clés de la région dans le but d'accorder une place plus grande à l'analyse sexospécifique dans leurs propres cadres de suivi institutionnels.

\section{Les systèmes d'alerte précoce dans le cadre de la surveillance de la sécurité alimentaire et nutritionnelle}

Les SAP-SAN permettent d'éclairer la planification des efforts de prévention et de gestion des crises alimentaires. Ils analysent les domaines de vulnérabilité d'une zone géographique et d'une population données en matière d'insécurité alimentaire et nutritionnelle au regard de toute une gamme de facteurs susceptibles d'influer sur cette vulnérabilité et d'indicateurs. Ils permettent de recueillir et d'analyser des informations primaires et secondaires afin de renforcer la prévention et les interventions précoces, essentiellement avant l'apparition d'une crise alimentaire. Ils peuvent être également utilisés au cours et dans le prolongement de ces crises pour en atténuer les répercussions et prévenir leur résurgence. Promouvoir le dialogue et la coordination, construire une vision cohérente et consensuelle de la situation alimentaire et nutritionnelle et nourrir ainsi la prise de décision : c'est le cœur de la mission du Réseau de prévention des crises alimentaires (RPCA) depuis près de trente-cinq ans au Sahel et en Afrique de l'Ouest. Créé en 1984, le Réseau est placé sous le leadership politique de la Communauté économique des États de l'Afrique de l'Ouest (CEDEAO) et de l'Union économique et monétaire ouest-africaine (UEMOA). Le Comité permanent inter-États de lutte contre la sécheresse dans le Sahel (CILSS) et le Secrétariat du Club du Sahel et de l'Afrique de l'Ouest (CSAO/OCDE) assurent le bon déroulement de ses activités. Le CILSS est également chargé de la coordination des activités du PREGEC au sein du RPCA. Les réunions du PREGEC rassemblent des experts de la sécurité alimentaire et nutritionnelle, ainsi que d'autres parties prenantes, six fois par an, afin de déterminer les perspectives nationales et régionales en matière alimentaire et nutritionnelle ${ }^{2}$. Les réunions du RPCA 


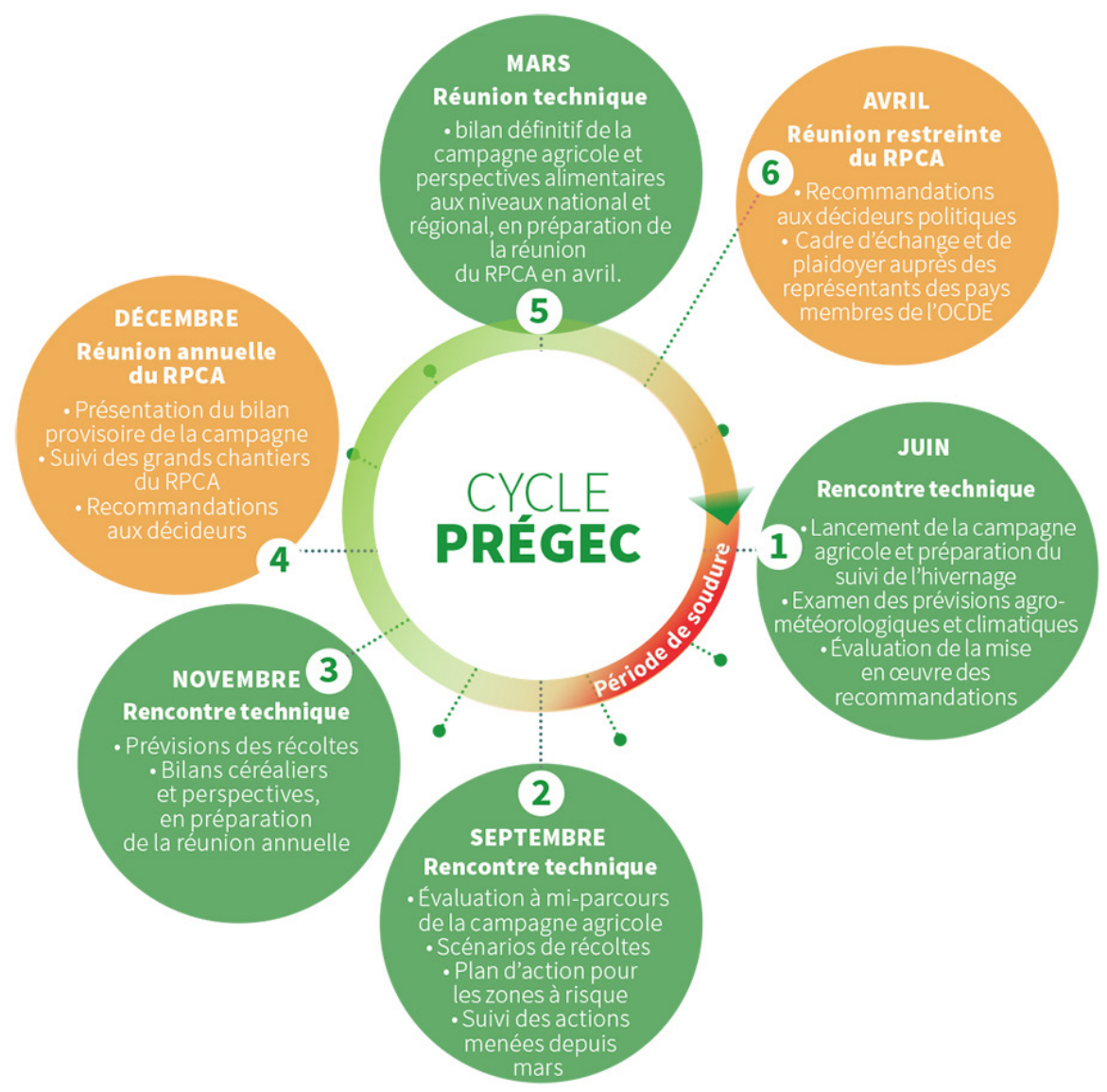

Source: RPCA

s'inscrivent dans ce cycle. Celui-ci est alimenté par divers outils et systèmes d'alerte précoce, dont des groupes de travail pluridisciplinaires (GTP) créés à l'initiative du CILSS, les bulletins d'alerte précoce du Réseau des systèmes d'alerte précoce contre la famine (FEWS NET) et du Système mondial d'information et d'alerte précoce sur l'alimentation et l'agriculture (SMIAR) de l'Organisation des Nations Unies pour l'alimentation et l'agriculture (FAO), ainsi que des bulletins d'alerte spéciaux établis sur la base de missions conjointes du CILSS, de la FAO, de FEWS NET, du Programme alimentaire mondial (PAM) et des gouvernements (CILSS, 2016).

Au nombre des membres du RPCA participant au PREGEC figurent des experts de la sécurité alimentaire et nutritionnelle d'Action contre la faim (ACF), de l'Agence française de développement (AFD), du CILSS, de la FAO, de FEWS NET, de l'Unité de soutien global du Cadre intégré de classification de la sécurité alimentaire (IPC), du Centre commun de recherche de 
la Commission européenne (CCR/CE), d'Oxfam, de Save the Children, du Fonds des Nations Unies pour l'enfance (UNICEF) et du PAM ${ }^{3}$ (tableau 1). Ces acteurs suivent une approche commune en matière de SAP-SAN édictée par le Cadre harmonisé. L'évolution technique des outils et des processus du Cadre a eu pour effet de renforcer les similitudes et la convergence avec l'IPC. Celui-ci soutient le Cadre harmonisé au moyen d'analyses pays, d'une consolidation régionale et d'un contrôle de la qualité, sur la base d'une feuille de route établie en commun ${ }^{4}$.

Tableau 1

Parties prenantes du Cadre harmonisé

\begin{tabular}{|c|c|c|}
\hline Échelon & Parties prenantes ${ }^{5}$ & Rôle principal \\
\hline National & $\begin{array}{l}\text { Responsables de l'action publique, société civile, } \\
\text { organismes professionnels, services techniques } \\
\text { nationaux (SAP, SAN, Système d'information sur } \\
\text { les marchés du bétail (LMIS), Système } \\
\text { d'information sur les marchés (SIM), Enquête } \\
\text { permanente agricole (EPA), etc.), partenaires } \\
\text { techniques et financiers et acteurs non étatiques } \\
\text { (ONG locales et internationales, organisations } \\
\text { d'agriculteurs, secteur privé, etc.), points de } \\
\text { contact/représentants nationaux. }\end{array}$ & $\begin{array}{l}\text { Acteurs à l'échelon local intervenant à toutes les } \\
\text { phases de la mise en œuvre du Cadre harmonisé }\end{array}$ \\
\hline Régional & CEDEAO, CILSS, UEMOA et ONG internationales & Soutien à la mise en œuvre du Cadre harmonisé \\
\hline International & $\begin{array}{l}\text { USAID, UE, AFD, FAO, PAM, UNICEF, FEWS NET, } \\
\text { Unité de soutien global de I'IPC, CCR/UE et ONG } \\
\text { internationales }\end{array}$ & $\begin{array}{l}\text { Direction des initiatives visant à prévenir et gérer les } \\
\text { crises alimentaires via des interventions de réhabilita- } \\
\text { tion d'urgence et par le renforcement de la résilience } \\
\text { des populations vulnérables. }\end{array}$ \\
\hline
\end{tabular}

Source: CILSS, 2016

Le Cadre harmonisé a été établi sur la base de recherches pluridisciplinaires menées par des experts de différentes institutions partenaires dans le but de : (i) mettre à jour les systèmes susceptibles de fournir les informations nécessaires à l'analyse des facteurs de vulnérabilité en matière de sécurité alimentaire et nutritionnelle au niveau des ménages, (ii) concevoir une méthodologie commune. La première version du Cadre harmonisé (1.0) a été diffusée en 2014 en tant qu'outil commun aux pays du Sahel et de l'Afrique de l'Ouest. Elle offre un cadre d'analyse complet pour les systèmes nationaux et régionaux de prévention et de gestion des crises alimentaires. Le Cadre aide les pays du CILSS et de la CEDEAO, ainsi que leurs partenaires, à analyser la sécurité alimentaire et nutritionnelle à l'échelon national et à différents niveaux d'administration, notamment le département, le comté ou le district. Les parties prenantes du Cadre harmonisé, qu'elles interviennent dans la mise en œuvre du manuel ou qu'elles en soient les bénéficiaires sont : i) nationales ; ii) régionales ; iii) internationales (CILSS, 2016). 
Le Cadre harmonisé définit des principes directeurs garantissant une mise en œuvre uniforme par l'ensemble des parties prenantes (CILSS, 2016) :

1. Ancrage institutionnel du Cadre harmonisé.

\section{Analyses objectives.}

3. Communication proactive des résultats du Cadre harmonisé.

En dehors des principes d'engagement des populations locales, d'inclusivité, de cohérence des politiques et de ciblage des plus vulnérables au sein d'une population donnée, le Cadre harmonisé ne donne aucune indication quant à l'utilisation de l'analyse sexospécifique ou des éléments des divers facteurs de vulnérabilité au sein d'une communauté pour soutenir les processus des SAP-SAN. Si le manuel du Cadre harmonisé mentionne à deux reprises le sexe comme variable susceptible d'avoir une influence, il le fait uniquement à propos des évaluations portant sur la nutrition. Les compétences ou les capacités en matière de problématique femmes-hommes sont également évoquées, mais les raisons de leur utilité ne font l'objet d'aucun examen. Surtout, aucune orientation claire n'est fournie sur la nécessité d'inclure des DVSA dans les registres de données des SAP-SAN. De même, le manuel ne contient pas d'instructions sur la façon d'utiliser ces données pour produire des statistiques ventilées par sexe et mener une analyse sexospécifique de la sécurité alimentaire et nutritionnelle. La diffusion d'une nouvelle version du Cadre harmonisé - le Cadre harmonisé 2.0 - est prévue. Cette mise à jour tiendra compte des nouvelles approches et contributions de divers acteurs et gouvernements, et donnera l'occasion de renforcer la prise en compte des disparités existant entre femmes et hommes dans les SAP-SAN de l'Afrique de l'Ouest.

\section{Les aspects sexospécifiques de la sécurité alimentaire et nutritionnelle}

L'égalité entre les sexes et l'autonomisation des femmes et des filles sont fondamentales pour garantir la sécurité alimentaire et nutritionnelle. Les rôles dévolus à chaque sexe sont des facteurs déterminants de la façon dont les individus et les ménages subissent et font face à l'insécurité alimentaire et nutritionnelle (CPI, 2017). Un SAP-SAN qui prend en compte les disparités entre femmes et hommes concourt à une planification sur une base factuelle adaptée aux différents besoins et capacités des populations. Il permet de mieux cibler les groupes les plus vulnérables. Des recherches menées par le service de recherche sur le genre et le développement de l'Institute of Development Studies, BRIDGE, dressent un panorama complet des façons dont la dynamique des rapports sociaux entre femmes et hommes peut favoriser la sécurité alimentaire ou, au contraire, lui nuire, et de la manière dont l'élaboration de programmes de sécurité alimentaire tenant compte 
des disparités entre les sexes peut contribuer à la justice sociale et à l'égalité (BRIDGE, 2014). BRIDGE explique clairement comment la dynamique des rapports sociaux entre les sexes influe sur la disponibilité des aliments, l'accès à la nourriture et son utilisation par les individus et les ménages, ainsi que sur les différentes façons dont l'insécurité alimentaire est vécue dans les situations d'urgence (PAM, 2016b). Ainsi, l'insécurité alimentaire est souvent liée au risque de violence sexiste, qui influe ensuite négativement sur l'accès à la nourriture (CPI, 2017). Une pénurie soudaine de nourriture au sein d'un voisinage sûr risque de contraindre les femmes et filles -en général chargées de l'approvisionnement en nourriture - à s'aventurer dans des zones plus difficiles, ce qui les isole et les expose à subir des actes de violence (CPI, 2017). De même, le manque de nourriture et d'autres ressources essentielles peut entraîner l'adoption de stratégies d'adaptation négatives, telles que le mariage précoce des jeunes filles, la violence conjugale et le commerce sexuel - autant de formes de violence qui ont un impact particulièrement fort et négatif sur les femmes et des filles. De même, les causes traditionnelles de la malnutrition sont intrinsèquement liées aux inégalités structurelles entre les sexes. Il est prouvé que l'autonomisation des femmes par l'exercice d'un pouvoir de décision a des répercussions positives sur la situation nutritionnelle globale des ménages.

\section{Les aspects sexospécifiques des systèmes d'alerte précoce pour la sécurité alimentaire et nutritionnelle}

La non-prise en compte par les SAP-SAN des rapports sociaux entre les sexes se répercute sur la conception des programmes sans considération des besoins spécifiques à chaque sexe. Le risque n'est pas uniquement un ciblage et une conception inefficientes des programmes, mais également une aggravation, en cas de crise (OSCE/ODIHR, 2009), des écarts préexistants entre les sexes et une exclusion plus grande encore des femmes de la conception des mesures préventives, ce qui accroît leur vulnérabilité face à un choc futur (CPI, 2017). Les besoins, difficultés et capacités différentes des femmes, des filles, des hommes et des garçons influent sur la disponibilité des aliments, leur accès à la nourriture et l'utilisation qu'elles et ils en font, et déterminent la façon dont elles et ils subissent les crises alimentaires. Comprendre ces dimensions contextuelles et sexospécifiques et éviter les stéréotypes sexistes (ACF, 2014) permet de mettre en place des efforts ciblés et économes de prévention, de réponse et d'assistance alimentaire pour les plus vulnérables.

Le tableau 2 est extrait d'un document de travail de 2015 de l'Overseas Development Institute (ODI) sur la problématique femmes-hommes et la résilience. Il illustre les liens spécifiques entre sexe, insécurité alimentaire et résilience face au changement climatique et aux catastrophes. 
Tableau 2

Exemples de liens entre problématique femmes-hommes, insécurité alimentaire et résilience

\begin{tabular}{|c|c|c|c|}
\hline Conséquences & Insécurité alimentaire & $\begin{array}{l}\text { Catastrophes : apparition } \\
\text { brutale }\end{array}$ & $\begin{array}{l}\text { Catastrophes : apparition progres- } \\
\text { sive }\end{array}$ \\
\hline Impacts & $\begin{array}{l}\text { Chute du rendement } \\
\text { Famine } \\
\text { Diminution des stocks halieu- } \\
\text { tiques } \\
\text { Magasins d'alimentation à la } \\
\text { merci d'épisodes extrêmes }\end{array}$ & $\begin{array}{l}\text { Inondations } \\
\text { Glissements de terrain } \\
\text { Tempêtes }\end{array}$ & $\begin{array}{l}\text { Sécheresses prolongées détruisant les } \\
\text { récoltes }\end{array}$ \\
\hline $\begin{array}{l}\text { Aspects } \\
\text { sexospéci- } \\
\text { fiques }\end{array}$ & $\begin{array}{l}\text { La production alimentaire } \\
\text { réclame davantage de temps et } \\
\text { d'énergie } \\
\text { Hausse de la charge de travail } \\
\text { des femmes, principales } \\
\text { responsables de la cuisine } \\
\text { Les femmes font pousser des } \\
\text { cultures ciblées pour les besoins } \\
\text { du foyer, sur des terres souvent } \\
\text { moins fertiles }\end{array}$ & $\begin{array}{l}\text { Les femmes, les hommes, les } \\
\text { filles ou les garçons peuvent } \\
\text { afficher des taux de mortalité } \\
\text { plus élevés en raison des } \\
\text { différents rôles dévolus à } \\
\text { chaque sexe, d'où découlent } \\
\text { une vulnérabilité et une } \\
\text { exposition aux risques } \\
\text { différents }\end{array}$ & $\begin{array}{l}\text { Les besoins et les capacités des } \\
\text { femmes sont souvent négligées } \\
\text { Les filles et les garçons abandonnent } \\
\text { l'école pour avoir un revenu }\end{array}$ \\
\hline $\begin{array}{l}\text { Stratégie de } \\
\text { survie/ } \\
\text { d'adaptation } \\
\text { sexospéci- } \\
\text { fiques }\end{array}$ & $\begin{array}{l}\text { Organisation de rations } \\
\text { Envoi des enfants auprès de } \\
\text { membres de leur famille } \\
\text { Migration des individus marginali- } \\
\text { sés } \\
\text { S'appuyer sur les programmes de } \\
\text { secours } \\
\text { Vente du bétail } \\
\text { Agroforesterie }\end{array}$ & $\begin{array}{l}\text { Dépendance à l'égard des } \\
\text { membres de la famille et de } \\
\text { l'aide des ONG }\end{array}$ & $\begin{array}{l}\text { Migration des individus marginalisés } \\
\text { Recours à la prière/S'appuyer sur la foi } \\
\text { religieuse }\end{array}$ \\
\hline
\end{tabular}

Source: Le Masson, V. et al 2015 
Le projet CASH+ mis en œuvre par la FAO au Sahel est un autre exemple dont les DVSA et l'analyse sexospécifique contribuent à l'instauration d'une approche axée sur les résultats. Il réaffirme le besoin de procéder à une analyse sexospécifique approfondie et de doter les systèmes de surveillance de la sécurité alimentaire et nutritionnelle d'indicateurs sexospécifiques. Ce projet applique une approche fondée sur la protection sociale (transferts en espèces et soutien à la production agricole) et cible expressément les femmes et les ménages dirigés par une femme. Le ciblage sur ces populations, basé sur des données concrètes, a renforcé la résilience des communautés en améliorant les stratégies d'adaptation, la nutrition (qualité et diversité) et les revenus (FAO, 2017d). L'intégration de dimensions sexospécifiques et de DVSA dans les systèmes de surveillance de la sécurité alimentaire et nutritionnelle, y compris dans les SAP, permet de montrer l'importance de la prise en compte de ces facteurs pour la mise en place de programmes de prévention et de gestion des crises qui ciblent efficacement les plus vulnérables.

L'intégration de l'analyse sexospécifique dans les SAP permet de déterminer de façon suivie les difficultés, les besoins, les capacités et les facteurs de vulnérabilité spécifiques à chaque sexe. Cela permet aussi de produire de l'information sur des réseaux de connaissance existants. Les rôles sociaux dans l'économie alimentaire et agricole, l'accès physique et financier des femmes aux marchés, le potentiel de génération de revenus, les types de produits alimentaires accessibles aux femmes et aux hommes respectivement, et l'exposition à la violence sexiste sont autant de variables importantes qui influent sur la résilience des individus et des ménages face à une crise alimentaire (El Rhomri, 2015). Par ailleurs, les systèmes spécifiques de connaissance des femmes et des hommes, ainsi que des jeunes, peuvent informer différemment les SAP. Une participation équilibrée des femmes et des hommes permet une planification mieux étayée et plus globale des mesures d'atténuation et de gestion des crises. À l'inverse, la mise à l'écart des femmes et des filles des consultations et des dialogues menés à l'échelon de la communauté limite la possibilité de tenir compte de leurs besoins et de leur vécu. Si certaines parties de la population sont exclues de la participation à la planification et à la mise en œuvre des SAP-SAN, ces systèmes risquent de ne pas pouvoir les protéger.

Les parties prenantes du PREGEC ont lancé des initiatives collectives visant à renforcer la prise en compte des disparités entre femmes et hommes dans les SAP-SAN. Celles-ci comprennent une mise à jour du Cadre harmonisé qui vise à garantir que les SAP puissent répondre à la diversité des défis et des situations que peuvent connaître les différentes populations qu'ils desservent. 


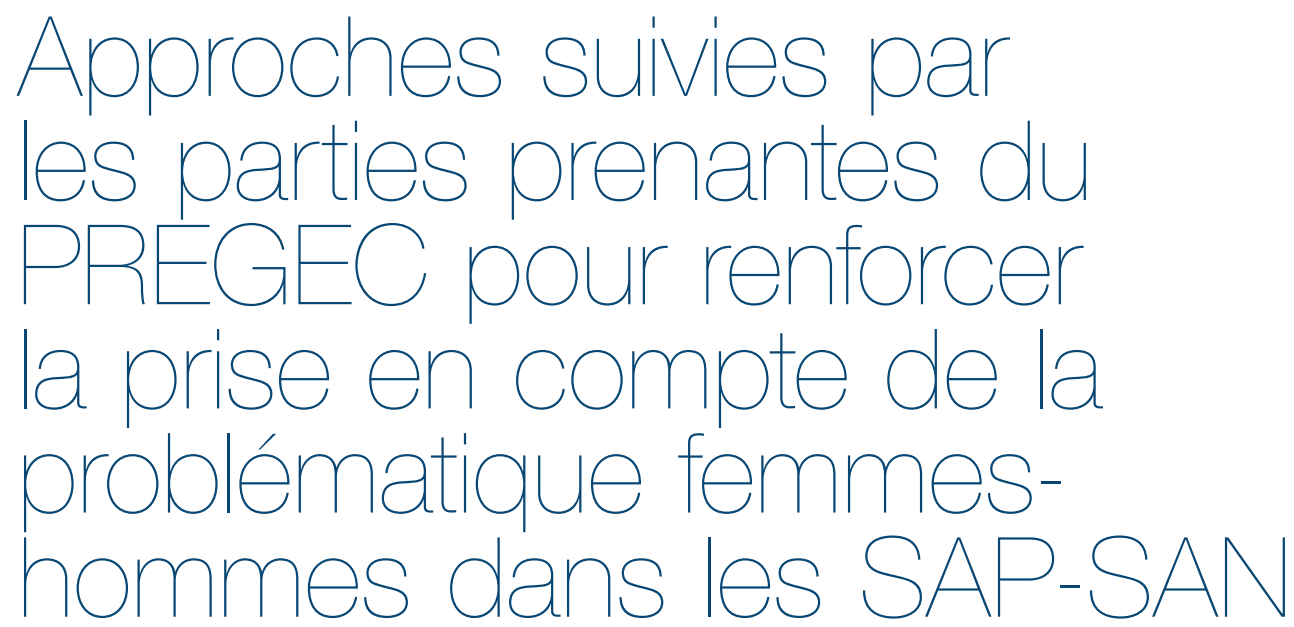

a plupart des organisations et des instances gouvernementales parties prenantes du PREGEC sont conscientes de l'importance de l'analyse sexospécifique et des DVSA pour la surveillance de la sécurité alimentaire et nutritionnelle ${ }^{6}$. Au cours de ces dernières années, plusieurs d'entre elles ont adopté des approches innovantes pour accélérer le renforcement des capacités en matière d'analyse sexospécifique. Mais ces parties prenantes ne sont pas encore en mesure d'assurer une intégration à grande échelle, systématique et durable de l'analyse sexospécifique dans les SAP-SAN régionaux et multipartites. Ce problème a pour effet d'amoindrir l'efficacité de la contribution que les systèmes d'alerte précoce apportent à la gestion des risques en situation d'urgence et à la recherche de l'équité dans la prévention et la gestion des crises.

Afin de mieux cerner les avancées réalisées et les lacunes qui subsistent, les efforts des membres du RPCA sont évalués sur base de trois critères :

1. L'ancrage de la surveillance de la sécurité alimentaire et nutritionnelle et des SAP dans les politiques institutionnelles en faveur de l'égalité femmeshommes (ou politiques équivalentes).

2. L'existence de cadres d'analyse, d'outils et de dispositifs de formation à l'appui de l'analyse sexospécifique dans les SAP-SAN.

3. La mise au point et l'utilisation de DVSA et d'indicateurs sexospécifiques pour les SAP-SAN. 
$\underline{\text { Tableau } 3}$

Parties prenantes du PREGEC ayant une politique en faveur de l'égalité femmes-hommes qui prend en compte la question des SAP-SAN

\begin{tabular}{|c|c|c|c|c|}
\hline $\begin{array}{l}\text { Institution/ } \\
\text { organisation }\end{array}$ & $\begin{array}{l}\text { Existence d'une } \\
\text { politique en faveur de } \\
\text { l'égalité femmes- } \\
\text { hommes }\end{array}$ & $\begin{array}{l}\text { Prise en compte de la } \\
\text { question de la } \\
\text { sécurité alimentaire et } \\
\text { nutritionnelle dans la } \\
\text { politique en faveur de } \\
\text { l'égalité femmes- } \\
\text { hommes }\end{array}$ & $\begin{array}{l}\text { Prise en compte de la } \\
\text { question de l'analyse } \\
\text { sexospécifique dans la } \\
\text { politique en faveur de } \\
\text { l'égalité femmes- } \\
\text { hommes }\end{array}$ & $\begin{array}{l}\text { Prise en compte de la } \\
\text { question des SAP-SAN } \\
\text { dans la politique en } \\
\text { faveur de l'égalité } \\
\text { femmes-hommes }\end{array}$ \\
\hline ACF & Oui & Oui & Oui & $\begin{array}{l}\text { Non, mais la prise en } \\
\text { compte de la probléma- } \\
\text { tique femmes-hommes } \\
\text { est évoquée dans la } \\
\text { préparation aux situa- } \\
\text { tions d'urgence }\end{array}$ \\
\hline CILSS & $\begin{array}{l}\text { Oui, mais elle n'est pas } \\
\text { encore prête pour } \\
\text { publication ; les } \\
\text { ressources financières } \\
\text { pour sa mise en œuvre } \\
\text { sont recherchées ; une } \\
\text { politique avait été établie } \\
\text { en 2008, mais elle est } \\
\text { aujourd'hui obsolète }\end{array}$ & $\begin{array}{l}\text { s.o. ; oui, dans la } \\
\text { politique en faveur de } \\
\text { l'égalité femmes- } \\
\text { hommes de } 2008\end{array}$ & $\begin{array}{l}\text { s.o. ; oui, dans la politique } \\
\text { en faveur de l'égalité } \\
\text { femmes-hommes de } 2008\end{array}$ & $\begin{array}{l}\text { s.o. ; absente de la } \\
\text { politique de } 2008\end{array}$ \\
\hline FAO & $\begin{array}{l}\text { Oui, mais n'a pas été } \\
\text { actualisée depuis } 2013\end{array}$ & Oui & Oui & $\begin{array}{l}\text { Non, mais complétée par } \\
\text { d'autres documents } \\
\text { d'orientation institution- } \\
\text { nels qui traitent de la } \\
\text { problématique femmes- } \\
\text { hommes dans le cas des } \\
\text { SAP-SAN }\end{array}$ \\
\hline FEWS NET & $\begin{array}{l}\text { Non, mais des pourpar- } \\
\text { lers sont en cours pour } \\
\text { amorcer son élaboration }\end{array}$ & s.o. & S.o. & s.o. \\
\hline OCHA & Oui & $\begin{array}{l}\text { Non, mais alignement } \\
\text { sur le Guide des genres } \\
\text { pour les actions } \\
\text { humanitaires du CPI }\end{array}$ & Oui & $\begin{array}{l}\text { Non, mais prise en } \\
\text { compte dans une } \\
\text { certaine mesure à travers } \\
\text { le Guide des genres pour } \\
\text { les actions humanitaires } \\
\text { du CPI }\end{array}$ \\
\hline
\end{tabular}




\begin{tabular}{|c|c|c|c|c|}
\hline Oxfam & Oui & $\begin{array}{l}\text { Non, mais la politique } \\
\text { est succincte et } \\
\text { complétée par des outils } \\
\text { et des ressources sur } \\
\text { l'analyse sexospécifique }\end{array}$ & $\begin{array}{l}\text { Non, mais la politique est } \\
\text { succincte et complétée par } \\
\text { des outils et des ressourc- } \\
\text { es sur la sécurité alimen- } \\
\text { taire et nutritionnelle }\end{array}$ & $\begin{array}{l}\text { Non, mais la politique est } \\
\text { succincte et complétée } \\
\text { par des études sur les } \\
\text { aspects sexospécifiques } \\
\text { de la sécurité alimentaire } \\
\text { et nutritionnelle et des } \\
\text { systèmes d'alerte } \\
\text { précoce concernant le } \\
\text { climat }\end{array}$ \\
\hline $\begin{array}{l}\text { Save the } \\
\text { Children }\end{array}$ & Oui & $\begin{array}{l}\text { Oui, mais la nutrition } \\
\text { seulement et non la } \\
\text { sécurité alimentaire }\end{array}$ & Oui & Non \\
\hline UNICEF & $\begin{array}{l}\text { Oui, sous la forme d'un } \\
\text { Plan d'action mondial } \\
\text { pour l'égalité femmes- } \\
\text { hommes }\end{array}$ & $\begin{array}{l}\text { Oui, mais la nutrition } \\
\text { seulement et non la } \\
\text { sécurité alimentaire }\end{array}$ & Oui & Non \\
\hline PAM & Oui & Oui & Oui & $\begin{array}{l}\text { Non, mais la question est } \\
\text { traitée dans une certaine } \\
\text { mesure dans la boîte à } \\
\text { outils sur la probléma- } \\
\text { tique femmes-hommes } \\
\text { associée à la politique }\end{array}$ \\
\hline
\end{tabular}

\section{Politiques en faveur de l'égalité femmes-hommes et ancrage institutionnel}

Les documents institutionnels sur l'action à mener pour promouvoir l'égalité entre les femmes et les hommes donnent aux acteurs concernés une idée de la façon dont l'analyse sexospécifique doit être intégrée dans toutes les étapes du cycle des programmes, ainsi que des moyens d'évaluation. L'élaboration d'une politique en faveur de l'égalité entre les sexes permet à l'organisation qui s'engage dans cette démarche d'expliciter l'approche qui va étayer la programmation d'activités propres à transformer les rapports sociaux entre les sexes, notamment la manière dont la collecte de DVSA et l'analyse sexospécifique, ainsi que les rapports qui en rendront compte, seront standardisés pour toutes les activités et toutes les étapes de la programmation.

Le processus du Cadre harmonisé est fondé sur l'initiative des autorités nationales qui agissent en partenariat conformément à l'un des principes directeurs du Cadre : l'ancrage institutionnel (CILSS, 2016). Des orientations claires, un effort d'impulsion et une forte résolution sont requises de la part des organisations et des instances gouvernementales participantes afin d'intégrer de manière constructive un dispositif d'analyse sexospécifique dans le Cadre harmonisé. 
Chaque partie prenante du PREGEC (ACF, FAO, Oxfam, Save the Children, PAM et UNICEF) s'est dotée d'une politique mondiale en faveur de l'égalité femmes-hommes ou d'un dispositif institutionnel équivalent, qui la guide dans la conception, à partir de l'analyse sexospécifique, de stratégies de programmation qui visent à transformer les rapports sociaux entre les femmes et les hommes. De plus, les politiques adoptées par ACF, la FAO, Oxfam, Save the Children et le PAM sont expressément conçues pour offrir des outils et des règles sur lesquelles s'appuyer pour intégrer la problématique femmes-hommes dans les SAP-SAN. FEWS NET et le CILSS sont en train de mettre au point des politiques en faveur de l'égalité entre les sexes ${ }^{7}$.

Élaborée à la lumière du Guide des genres pour les actions humanitaires du Comité permanent interorganisations (CPI), la politique d'ACF en faveur de l'égalité femmes-hommes constitue un bon exemple de cadre institutionnel contenant des indications concrètes sur l'application de l'analyse sexospécifique pour la surveillance de la sécurité alimentaire et nutritionnelle, ainsi que pour la préparation et la réponse aux situations d'urgence. Elle est diffusée dans tous les secteurs et complétée par une boîte à outils qui explique comment effectuer une analyse sexospécifique dans le cadre d'un SAP-SAN et utiliser les résultats de celle-ci pour éclairer les interventions humanitaires (ACF, 2014).

S'il est important de disposer d'une politique forte de promotion de l'égalité entre les femmes et les hommes, celle-ci ne sera efficace que si elle est bien mise en œuvre. Il est difficile de déterminer dans quelle mesure certains aspects d'une politique en l'occurrence, la réalisation d'une analyse sexospécifique dans le cadre des SAP-SAN sont compris et mis en pratique.

Même dans le cas des organisations ayant une politique en faveur de l'égalité des sexes qui prévoit des outils et des méthodes pour la prise en compte de la problématique femmes-hommes, il n'est pas possible de déterminer si un décalage persiste entre ce que cette politique exige en matière d'analyse sexospécifique dans le cadre des SAP-SAN et ce qui est fait concrètement. C'est ce qui ressort d'un document d'Oxfam, organisation qui a un solide programme d'action mondial en faveur de l'égalité femmes-hommes et qui a indiqué que le manque de DVSA et l'absence d'analyse sexospécifique avait empêché la conduite d'interventions humanitaires suffisamment adaptées lors de la crise alimentaire somalienne de 2017 (Feeny, 2017). Les normes minimales pour la prise en compte de la problématique femmes-hommes dans les situations d'urgence, qui prévoient l'utilisation de DVSA et la réalisation d'une analyse sexospécifique afin d'éclairer la programmation, n'ont pas été appliquées dans ce cas. La politique de la FAO en matière d'égalité entre les sexes comporte des orientations sur les SAP-SAN sensibles aux disparités entre femmes et hommes, mais les consultations avec les partenaires n'ont pas permis de savoir précisément dans quelle mesure cette politique est connue du personnel présent dans la région, et si les boîtes à outils sur le sujet sont suffisamment utilisées ${ }^{8}$. 


\section{Cadres d'analyse, outils et formation}

Si une politique en faveur de l'égalité femmes-hommes engage l'organisation qui s'en dote à se fixer des objectifs en la matière, et rend le personnel et les partenaires de celle-ci responsables vis-à-vis de ces objectifs, il faut toutefois noter que ces acteurs seront dans l'impossibilité de les atteindre en l'absence de renforcement des capacités adapté et de grande envergure. Un cadre d'analyse sexospécifique solide et bien conçu, complété par des outils et du renforcement des capacités permettra d'effectuer des travaux de qualité dans une optique stratégique et éclairer la prévention et la gestion des risques dans les situations d'urgence.

Dans le cas des parties prenantes du PREGEC qui possèdent une politique en faveur de l'égalité femmes-hommes, les engagements sont généralement assortis d'orientations et de cadres qui précisent comment effectuer de l'analyse sexospécifique. De plus, la plupart d'entre elles peuvent s'appuyer sur des plateformes mondiales d'aide au renforcement des capacités. Par exemple, le CPI, la banque de données de la Banque mondiale et la Division de la statistique des Nations unies, entre autres, fournissent des outils et des orientations sur l'analyse sexospécifique pour les systèmes de surveillance de la sécurité alimentaire et nutritionnelle. Ceci comprend les systèmes d'alerte précoce, mais dans une moindre mesure. Le Guide des genres pour les actions humanitaires du CPI (CPI, 2017) présente un large éventail d'outils, de mécanismes et d'approches pour l'intégration de l'analyse sexospécifique dans l'action humanitaire pour tout un éventail de secteurs, dont la sécurité alimentaire et la nutrition. S'il ne traite pas expressément des systèmes d'alerte précoce, une grande part des orientations et des ressources qu'il expose au sujet de l'analyse sexospécifique sont applicables à ces systèmes, ainsi qu'aux interventions concernant la sécurité alimentaire et la nutrition.

La FAO et le PAM ont publié un certain nombre d'outils et autres documents conjoints importants sur l'analyse sexospécifique pour la surveillance de la sécurité alimentaire et nutritionnelle. En 2008, ils ont fait paraître un ouvrage intitulé Socio-Economic and Gender Analysis (SEAGA) for Emergency and Rehabilitation Programmes. Celui-ci présente douze modules portant sur la prise en compte de la problématique femmes-hommes pour éclairer les interventions d'urgence et aider les spécialistes à inscrire la planification dans la recherche de transformations propices à l'égalité entre femmes et hommes. L'outil d'analyse socioéconomique et sexospécifique dont il est question renseigne sur la façon dont il faut procéder pour recueillir des DVSA et effectuer une analyse sexospécifique pour les besoins du suivi des interventions d'urgence. Parmi les indicateurs recommandés figurent l'aptitude à lire, écrire et compter, les déplacements/migrations, les situations de conflit et les stratégies d'adaptation (FAO et PAM, 2008). De plus, une formation interactive en ligne est proposée par la FAO et le PAM afin de compléter la publication sur l'analyse socioéconomique et sexospécifique. Cette formation demeure accessible au personnel et aux partenaires. 
S'appuyant sur sa politique mondiale en faveur de l'égalité femmes-hommes, le Bureau régional du PAM pour l'Afrique de l'Ouest et du Centre a élaboré et mis en œuvre un dispositif de formation à l'analyse sexospécifique pour la sécurité alimentaire et nutritionnelle. Ce dispositif est expérimenté par le personnel du Bureau régional et des services centraux du PAM. Ceci implique que le personnel technique responsable de l'analyse sexospécifique et celui chargé de l'analyse de la vulnérabilité ont pu expérimenter le dispositif. Il a également été testé par le personnel technique au Ghana et en Guinée en 2017 et 2018, et est maintenant mis en œuvre dans toute la région9 . Le contenu du dispositif de formation est fondé sur une documentation sur l'analyse sexospécifique, élaborée au niveau des instances mondiales du PAM dans le but de prêter appui à l'Unité d'analyse et de cartographie de la vulnérabilité (VAM), ainsi que sur la boîte à outils du PAM relative à la problématique hommes-femmes, publiée en 2017. Alignée sur la politique du PAM en matière de problématique hommes-femmes, cette boîte comporte des chapitres sur l'analyse sexospécifique, le suivi, l'évaluation et l'analyse de la sécurité alimentaire et de la vulnérabilité ${ }^{10}$. Le dispositif de formation mis en place en Afrique de l'Est et du Centre ne traite pas des SAP, et le dossier documentaire du PAM sur l'analyse sexospécifique et sa boîte à outils fournissent très peu d'indications sur l'analyse sexospécifique dans le cadre des SAP en particulier.

En 2016, la FAO a élaboré un guide de formation spécial sur les SAP-SAN intitulé Gender-responsive Disaster Risk Reduction (DRR) in the Agriculture Sector. Ces lignes directrices, inspirées du Cadre de Sendai (UNISDR, 2015), attirent l'attention sur le rôle différent que jouent les femmes, les filles, les hommes et les garçons dans la réduction des risques de catastrophe et le renforcement de la résilience, ainsi que sur les facteurs de vulnérabilité qui les touchent respectivement. Le guide fournit des outils et des méthodes pour la planification d'activités propres à transformer profondément les rapports sociaux entre femmes et hommes, la collecte de DVSA et l'analyse sexospécifique. Élaboré dans une optique mondiale, il pourrait être adapté en vue d'être appliqué dans d'autres domaines que la réduction des risques de catastrophe et dans les pays couverts par le cycle du PREGEC.

Dans la Stratégie conjointe de la FAO et du PAM en matière de systèmes d'information sur la sécurité alimentaire et nutritionnelle, dont les SAP constituent une fonction, il est indiqué que l'analyse sexospécifique est essentielle à la surveillance de la sécurité alimentaire et nutritionnelle, et que la prise en compte des inégalités entre femmes et hommes sur le plan de la sécurité alimentaire est une obligation. Les informations recueillies par les SAP citées dans la Stratégie conjointe, notamment le Système mondial d'information et d'alerte rapide sur l'alimentation et l'agriculture (SMIAR) et le Système de prévention et de réponse précoce contre les ravageurs et les maladies transfrontières des animaux et des plantes (EMPRES), sont incorporées dans le rapport mondial de la FAO sur l'alerte et l'intervention précoces. 


\section{Tableau 4}

Politique en matière de genre et Boîte à outils Genre d'ACF : recommandations concernant la collecte de DVSA et leur diffusion

\section{Recommandations essentielles d'ACF pour garantir une collecte et une utilisation appropriées des données ventilées par sexe et par âge (DVSA)}

- Ajouter des indicateurs pertinents en matière de sexe et d'âge aux enquêtes et aux systèmes de suivi.

- La collecte et l'analyse de données en situation d'urgence peuvent être difficiles ; I'utilisation de sources existantes peut aider à identifier les problématiques de genre et à développer des points de comparaison pour les indicateurs. II convient d'examiner la littérature et les données existantes afin de déterminer les différents rôles des hommes, des femmes, des personnes âgées, des adultes, des jeunes et des enfants dans les secteurs clés avant la crise. C'est particulièrement utile pour avoir un point de comparaison à partir duquel extrapoler une fois que les données de l'évaluation initiale sont disponibles. Par ailleurs, cela permet de mieux identifier les groupes les plus touchés. Exemples : les enquêtes démographiques et sanitaires (EDS), les rapports nationaux du Comité pour l'élimination de la discrimination à l'égard des femmes (CEDAW), les données nationales de l'Indice pour l'égalité des sexes, etc.

- Examiner les études et les rapports clés portant sur les groupes vulnérables ou sur les risques/menaces spécifiques préexistantes au sein de la population concernée.

- Examiner les cadres légaux ou coutumiers pour identifier les domaines potentiels de discriminations fondées sur le genre ou sur l'âge.

- Veiller à ce que les équipes responsables des évaluations (les évaluateurs et les traducteurs) soient composées d'hommes et de femmes. Trouver un compromis réfléchi entre la technicité des équipes (par ex. avec des agronomes, des nutritionnistes, des vétérinaires et des agents de santé clinique) et leur diversité (pour leur permettre de se relier à tous les groupes de la communauté).

- Organiser des entretiens et des groupes de discussion femmes-hommes séparés en fonction des âges et des positions socioéconomiques.

- Veiller à ce que les informatrices clés soient interviewées (notamment les dirigeantes communautaires, les sagesfemmes, les infirmières, les femmes en position de force sur le marché et les institutrices) ; elles connaissent souvent les besoins immédiats des femmes.

- Lorsqu'un groupe parle au nom d'un autre et fait des suppositions quant à l'accès de ce dernier aux services, essayer de trianguler ces informations avec le groupe concerné ou, lorsque cela n'est pas possible (comme pour les nourrissons, par exemple), essayer d'identifier les meilleurs informateurs possibles.

- Veiller à ce que les DVSA concernant tous les informateurs clés, les individus et les membres du foyer soient enregistrées afin:

- D'évaluer si des parties importantes de la population dont les avis seraient nécessaires pour développer des réponses appropriées n'ont pas été consultées et;

- De déterminer s'il existe des différences importantes entre les membres des populations les plus vulnérables (quels que soient leur sexe et leur âge) en termes de besoins et de capacité d'accès aux services de base.

- Veiller à ce que les membres des équipes formulent des observations sur les effets sur l'infrastructure et l'impact sur les populations civiles, en indiquant où l'accès aux services est le plus difficile (voire bloqué) pour certains segments de la population.

- Lorsque les individus s'inscrivent aux distributions, veiller à ce que les données relevées incluent le sexe et l'âge (ou la tranche d'âge) de chaque personne.

- Synthétiser les données de manière cohérente en avançant des recommandations factuelles puis transmettre ces rapports aux personnes chargées de la prise de décision. 


\section{Tableau 5}

Indicateurs sexospécifiques du PAM recommandés pour l'analyse de la sécurité alimentaire

\section{Indicateurs sexospécifiques sur l'accès aux aliments}

- Pouvoir d'achat des ménages déterminé d'après leur revenu, selon le sexe du chef du ménage.

- Gains moyens des ménages dirigés par une femme en pourcentage des gains moyens des ménages dirigés par un homme.

- Différences d'accès aux actifs productifs (ou de maîtrise de ces actifs) entre les ménages dirigés par un homme et les ménages dirigés par une femme.

- Participation aux comités locaux alimentation-agriculture, par sexe et par âge.

- Actifs et épargne des ménages en période de difficulté, selon le sexe du chef du ménage.

- Nombre moyen de repas consommés au cours des sept derniers jours, par les femmes, les hommes, les filles et les garçons.

- Indice des stratégies de survie, selon le sexe du chef du ménage.

- Niveau d'instruction moyen des enfants/niveau atteint par les adules, ventilé par sexe.

- Part des dépenses consacrées à la nourriture dans le total des dépenses des ménages, selon le sexe du chef du ménage.

- Accès au crédit/aux marchés, selon le sexe du chef du ménage.

- Pourcentage de ménages dans lesquels tant les femmes que les hommes travaillent (et contribuent au revenu du ménage).

- Division du travail (rémunéré et non rémunéré) entre les différents membres du ménage et contraintes liées au temps, par sexe et par âge.

- Inclure aussi des statistiques sur les jeunes filles, population qui est en général entièrement engagée dans le travail de production et de reproduction mais qui tend à être insuffisamment comptabilisée et sous-estimée, d'où des interventions inadaptées (BRIDGE 2014).

\section{Indicateurs sexospécifiques sur l'utilisation des aliments}

- Prévalence des enfants de moins de cinq ans ayant un retard de croissance/atteints de cachexie/présentant un déficit pondéral, par sexe.

- Prévalence des femmes non enceintes adultes/en âge de procréer qui sont légèrement/modérément/gravement sous-alimentées ou en surpoids.

- Prévalence de l'anémie par déficit en fer chez les femmes en âge de procréer et les enfants de moins de cinq ans, par sexe.

- Prévalence des maladies (fréquence moyenne), par sexe et par groupe d'âge.

- Taux de mortalité, par âge et par sexe.

- Périmètre brachial, ventilé par sexe, des enfants âgés de 12 à 59 mois et/ou IMC des femmes en âge de procréer.

\section{Indicateurs sexospécifiques sur la disponibilité des aliments}

- Production, entreposage, achats des ménages, selon le sexe du chef du ménage.

- Capacité des femmes et des hommes à posséder la terre, à en hériter et à en exercer concrètement la propriété.

- Proportion de femmes qui prennent part à des réunions politiques à l'échelle locale.

- Différences d'accès au crédit entre les ménages dirigés par un homme et les ménages dirigés par une femme.

- Pourcentage de femmes et d'hommes employés dans différents secteurs. 


\section{Indicateurs sexospécifiques sur les situations d'urgence}

- Répartition de la population cible, par sexe et par groupe d'âge (au niveau de l'individu et/ou du chef du ménage).

- Proportion d'enfants en déficit pondéral parmi les enfants touchés âgés de 24 à 59 mois, par sexe.

- Proportion de filles parmi les enfants affectés en âge de fréquenter l'école primaire non scolarisés.

- Nombre et lieu d'habitation des personnes (femmes, hommes, filles et garçons) ayant subi des violences sexistes.

- *Utiliser la base de données du GBVIMS ou d'autres sources de données secondaires.

- Pourcentage le plus récent de la population touchée qui a un faible score de consommation alimentaire (SCA)/qui est en situation de sous-alimentation, selon le sexe du chef du ménage.

- Taux le plus récent de retard de croissance/de cachexie chez les filles et les garçons.

- Estimation la plus récente de la part moyenne des dépenses alimentaires des ménages dans le total de leurs dépenses, selon le sexe du chef du ménage.

- Indice des stratégies de survie, selon le sexe du chef du ménage.

Source: PAM 2016b

\section{Tableau 6}

Politique et Boîte à outils en matière de genre d'ACF: exemples de questions pour l'analyse sexospécifique

\section{Exemples de questions pour le secteur de la nutrition}

- Quelles sont les différences entre les rôles et les responsabilités des femmes (jeunes ou âgées) et des hommes (jeunes ou âgés) en termes: a) d'accès aux services de santé pour le traitement de la malnutrition aiguë ; b) d'influence en matière de pratiques d'allaitement exclusif ; c) d'influence en matière de pratiques d'alimentation complémentaire ; d) d'accès aux aliments nutritifs (fruits, légumes, etc.) ?

- Quelles sont les habitudes d'alimentation au sein du foyer (c'est-à-dire qui mange en premier, les enfants ou le père, etc.) ?

- Qui décide (et de quelle manière) si les récoltes vont être consommées ou vendues (légumes, céréales, viande, poisson, etc., selon le contexte) ?

- Les données (ventilées par sexe et par âge) concernant le statut nutritionnel des populations indiquent-elles que les filles ou les garçons sont affecté(e)s de façon disproportionnée ? Si oui, comment expliquer ces différences ?

- Quels sont les niveaux d'anémie parmi les femmes en âge de procréer (données nationales ou issues de groupes spécifiques en fonction du programme) ?

- Quelles sont les pratiques et les tabous socioculturels et/ou les croyances culturelles en termes de : a) pratiques de soins ; b) régimes des femmes enceintes ou allaitantes ; c) utilisation de colostrum ; d) allaitement exclusif ; e) sevrage des nourrissons après six mois d'allaitement exclusif (etc.) ?

- De quelles manières ces pratiques peuvent-elles affecter le statut nutritionnel des femmes, filles, garçons et hommes?

- Comment les garçons et les hommes organisent-ils leurs repas quotidiens lorsqu'ils sont loin de chez eux (à savoir lors de migrations saisonnières pour le travail, lors de périodes d'insécurité, etc.) ? (Cuisinent-ils eux-mêmes ? Font-ils appel à des personnes extérieures pour cuisiner ? Si oui, les paient-ils ? Est-ce qu'ils savent cuisiner mais refusent de le faire car ce n'est pas 'culturellement' accepté ? etc.)

- Lorsque les femmes sont chefs de famille (ventiler les données par âge), ont-elles accès à de la nourriture nutritive en quantité suffisante (à définir selon le contexte)?

- De quelle manière les femmes et les hommes âgés accèdent-ils à la nourriture, et la nourriture distribuée satisfait-elle leurs besoins spécifiques? 


\section{Exemples de questions concernant le secteur de la sécurité alimentaire et des moyens d'existence}

- De quelle manière la nourriture est-elle préparée et partagée en famille ?

- Les femmes, les filles, les garçons et les hommes ont-elles/ils un accès égal au marché local, aux opportunités professionnelles, aux intrants, aux services ainsi qu'aux biens tels que le bétail ou les terres?

- Qui reçoit l'assistance (en espèces ou en nature) au nom du foyer ? Qui décide de la façon de l'utiliser?

- Qui produit/achète/mange tel ou tel type de nourriture ? Qui mange en premier ?

- Les femmes, les filles, les hommes et les garçons sont-elles/ils affecté(e)s de manière identique par la famine saisonnière ? Existe-t-il des différences dans leur manière de lutter contre la faim saisonnière ?

- Les femmes doivent-elles effectuer une journée « double », voire « triple » exécuter les tâches ménagères, préparer la nourriture, s'occuper des enfants et des personnes âgées, effectuer des travaux agricoles ou autres ?

- Les femmes, les filles, les hommes et les garçons adoptent-elles/ils des stratégies d'adaptation différentes ?

Source: ACF 2014

Ce rapport ( traduit des avertissements en actions d'anticipation ») en analysant les informations actualisées sur les prévisions et en formulant des recommandations visant à une intervention rapide pour chacun des pays en situation d'insécurité alimentaire qui sont ciblés. Si la place faite à l'analyse sexospécifique dans les rapports mondiaux de la FAO sur l'alerte et l'intervention précoces est moindre par rapport à la Stratégie conjointe, il existe néanmoins des exemples positifs. Ainsi, le rapport mondial de juin 2017 recommande, dans le cas du Nigeria, de soutenir les éleveurs vulnérables en visant tout particulièrement les ménages dirigés par une femme et possédant des petits ruminants, en raison de considérations touchant à la problématique femmes-hommes que la surveillance effectuée dans le cadre du SAR rapide a fait apparaître.

L'Initiative régionale « Genre et marchés ») mise en place par le PAM en Afrique de l'Ouest fait appel à plusieurs outils innovants et donne lieu à la rédaction de rapports utiles sur les aspects sexospécifiques de la sécurité alimentaire. Tous les documents qui s'y rapportent sont rendus publics dans le but de diffuser les connaissances sur les diverses activités des marchés et des chaînes de valeur des produits alimentaires qui touchent à la problématique femmes-hommes, ainsi que sur les outils et la formation liés à l'analyse sexospécifique à effectuer dans le cadre de la surveillance des marchés pour la sécurité alimentaire et nutritionnelle. L'équipe du PAM a aussi conçu des modules de formation à l'analyse des marchés sous l'angle de la problématique femmes-hommes à l'intention du personnel et des partenaires de toute la région, aux fins de la conception et de la mise en œuvre des SAP-SAN ${ }^{11}$.

Par ailleurs, l'équipe du PAM chargée de l'initiative Genre et marchés est en train de mettre au point un indice qui permettra de réunir des données et autres informations sur les aspects de l'autonomisation des femmes qui touchent aux marchés et aux chaînes de valeur des produits alimentaires. Pour ce faire, elle s'appuie sur le cadre relatif à l'Indice d'autonomisation des 
femmes dans l'agriculture (WEAI)(IFPRI, 2012), mis au point par l'IFPRI et USAID, qui prend en compte les normes sociales et culturelles sexuées déterminant le rôle et le vécu des femmes dans le domaine de l'agriculture. Le BIT et l'IFPRI ont aussi entrepris de définir des approches analogues pour d'autres secteurs que l'agriculture. L'avantage le plus important que présentent des cadres comme celui du WEAI est aussi leur principal inconvénient : ils sont à la fois très complets et très instructifs, mais ils exigent également beaucoup de moyens et de ressources pour la réalisation d'enquêtes. L'équipe du PAM chargée de l'Initiative Genre et marchés a adapté le WEAI simplifié afin de faciliter la réalisation de plusieurs études de cas en Afrique de l'Ouest, ce qui représente un exemple intéressant d'approche consistant à modifier la conception d'un outil en fonction des besoins d'une organisation et des ressources dont elle dispose. Des indices tels que le WEAI offrent un cadre utile et accessible car ils permettent de savoir précisément quels indicateurs et quelles statistiques recueillir, analyser et décrire pour éclairer la dynamique des disparités femmes-hommes. Bien que les parties prenantes du PREGEC disposent d'une large palette de ressources, celles-ci n'ont pas été utilisées pour apporter au cycle du PREGEC ou au manuel du Cadre harmonisé des éléments qui permettent d'assurer une meilleure prise en compte de la problématique femmes-hommes dans les SAP-SAN.

\section{Données ventilées par sexe et par âge (DVSA) et indicateurs sexospécifiques}

La ventilation par sexe et par âge des données sur les personnes constitue l'opération de base à effectuer pour garantir que l'action menée auprès d'une population sera équitable et ciblée, et qu'elle ne fera pas d'exclus. Ces données, qui peuvent être recueillies au moyen de méthodes quantitatives et/ou qualitatives, permettent d'analyser la dynamique du pouvoir, le rôle respectif des femmes et des hommes et les disparités liées au sexe et à l'âge qui ont une influence sur la vulnérabilité (PAM, 2016d). La majorité des parties prenantes du PREGEC recueillent des DVSA, mais elles ne procèdent pas toujours de manière uniforme ; les chiffres peuvent être insuffisamment disséminés et, dans bien des cas, il aucune analyse sexospécifique n'est réalisée. De plus, les données primaires utilisées pour les SAP en Afrique de l'Ouest proviennent en grande partie des données nationales fournies par les ministères et les services de statistiques, où la collecte et la communication de DVSA sont relativement limitées. Il est donc difficile de s'assurer que ces données, lorsqu'elles sont recueillies, sont prises en compte dans le cycle du PREGEC.

Certaines parties prenantes du PREGEC apportent une contribution non négligeable à la production de DVSA et d'indicateurs sexospécifiques.

- L'équipe mondiale du PAM chargée de l'analyse et de la cartographie de la vulnérabilité (VAM) recueille des DVSA lors d'enquêtes sur les ménages, auxquelles elle ajoute dans certains cas des questions sur la 
situation respective des femmes et de hommes en ce qui concerne, par exemple, l'accès aux ressources productives agricoles et la maîtrise de ces ressources. Ces données sont généralement codées et publiées sur le site internet de l'équipe VAM du PAM ${ }^{12}$.

- L'Approche consolidée du PAM pour le compte rendu des indicateurs de la sécurité alimentaire (CARI) est une méthode qui sert à classer les ménages selon leur situation sur le plan de la sécurité alimentaire, en ventilant les données selon le sexe du chef du ménage (PAM, 2015), et qui permet notamment de calculer le score de consommation alimentaire et la part des dépenses alimentaires, ainsi que de déterminer les stratégies de survie fondées sur les moyens de subsistance. La méthode CARI peut donc être utile pour établir des DVSA et construire des indicateurs sexospécifiques. Néanmoins, elle n'est pas prise en compte dans le Cadre harmonisé.

- La FAO recueille et publie des DVSA et des indicateurs sexospécifiques sur le secteur agricole. Elle gère aussi la base de données Genre et le droit à la terre, qui contient des DVSA et des indicateurs sur les exploitants agricoles et les propriétaires fonciers, ainsi que sur la répartition de la superficie des terres agricoles et la valeur de celles-ci (FAO, s.d.-c). Dans le cas de l'Afrique de l'Ouest, ces données sont actuellement disponibles pour le Burkina Faso, la Côte d'Ivoire, la Guinée, le Mali, le Nigeria et le Sénégal.

- La Boîte à outils Genre d'ACF contient des recommandations et des orientations pour collecter des DVSA de bonne qualité et pour augmenter leur utilisation.

Il est essentiel de disposer de données secondaires pour pouvoir informer les DVSA, mais des problèmes se posent lorsque les sources dont proviennent ces données ne sont pas très connues ou accessibles au public (PAM, 2016c). Afin d'y remédier, le Bureau du PAM pour l'Afrique de l'Ouest a procédé, en 2016, à l'examen de données secondaires issues d'analyses sur la problématique femmes-hommes et les marchés de la région. L'un des principaux résultats de cet exercice a été l'établissement d'une liste de sources de données secondaires utiles pour l'étude de la problématique femmes-hommes et des marchés, dont un tableau indiquant quelles données et indicateurs sont ventilés par sexe et par âge. Ce tableau recense les bases de données qui couvrent la problématique femmes-hommes dans les marchés du travail, les bases de données sur les taux de scolarisation et celles sur les droits fonciers. Il en ressort que des DVSA sont disponibles dans bien des cas, mais qu'aucune initiative n'a été prise pour les rassembler et les synthétiser de façon à constituer d'autres systèmes, comme des SAP interinstitutionnels. 
La collecte de DVSA et les rapports y afférents permettent d'élaborer des indicateurs sexospécifiques adaptés au contexte et à la réponse. Les orientations mondiales définies par le PAM dans le document intitulé Gender Standards on Food Security Analysis (WFP, 2016b)offrent une liste non exhaustive d'indicateurs sexospécifiques de la disponibilité des aliments, l'accès à ces derniers et leur utilisation, établis à partir de DVSA et de statistiques sur la problématique femmes-hommes. Le but est d'offrir aux acteurs concernés la possibilité de choisir les indicateurs les plus adaptés aux domaines sur lesquels portent leurs efforts de programmation.

Une fois les indicateurs sélectionnés, il faut déterminer la meilleure manière de les analyser. Partant des enseignements tirés de l'action menée en Somalie, Oxfam recommande de pondérer les indicateurs de manière à donner la priorité à ceux qui ont le plus d'importance du point de vue des femmes et des filles (Feeny, 2017). Des indices tels que le WEAI offrent un cadre souple pour pondérer les indicateurs en fonction de l'objectif de l'analyse et de la définition de concepts tels que celui d'(( autonomisation ), qui dépend du contexte (IFPRI, 2012).

Pour la réalisation d'une analyse qualitative plus étendue et la collecte d'informations plus complètes, la Politique et la Boîte à outils en matière de genre d'ACF incluent des exemples de questions (tableau 6) pour guider les évaluations sexospécifiques sur le secteur de la nutrition et celui de la sécurité alimentaire et des moyens d'existence. Ces questions peuvent aider les parties prenantes du PREGEC à structurer et cibler l'analyse sexospécifique en l'articulant autour des aspects de la dynamique de la problématique femmes-hommes à prendre en compte dans le cadre des SAP-SAN.

Selon les principales parties prenantes, un débat a lieu dans le cadre des réunions du PREGEC sur la nécessité de disposer de DVSA standardisées et d'intégrer l'analyse sexospécifique dans les dispositifs actuels. Un processus d'actualisation du Cadre harmonisé, version 1.0, a été engagé en vue de produire une version 2.0. D'après les entretiens qui ont eu lieu avec des organismes de premier plan, cette nouvelle version va probablement recommander la prise en compte de certains indicateurs sexospécifiques à inclure en tant que facteurs qui contribuent aux crises de sécurité alimentaire. Des spécialistes d'organismes parties prenantes ont été invités à apporter des éléments d'ordre technique pour l'élaboration des projets de la version $2.0 \mathrm{du}$ Cadre harmonisé13. Toutefois, il sera important de veiller à ce que l'analyse sexospécifique soit incorporée dans le processus de développement de cette mise à jour, et qu'elle ne soit pas abordée seulement une fois que le contenu fondamental du manuel aura été établi.

Les consultations organisées avec les principales parties prenantes du PREGEC ont montré qu'il existait un vaste consensus au sujet de l'importance de la prise en compte de la problématique femmes-hommes dans le domaine 
de la sécurité alimentaire et nutritionnelle et dans les SAP-SAN. En témoigne le fait que la majorité des partenaires ont mis en place une politique institutionnelle en faveur de l'égalité femmes-hommes, et qu'ils affectent des ressources supplémentaires à la mise en œuvre des objectifs de cette politique. De nouveaux cadres et outils d'analyse sexospécifique ont été et continuent d'être élaborés, afin de répondre à l'évolution de la demande d'informations sur la problématique femmes-hommes. Le mouvement qui se manifeste au niveau mondial en faveur de la production de données sur les disparités existant entre les sexes et de statistiques sexospécifiques est aussi très fructueux. Un abondant corpus de données secondaires sur différents aspects de la problématique femmes-hommes aux niveaux local, national, régional et mondial est désormais accessible au public. Malgré ce mouvement de fond, la version actuelle du Cadre harmonisé (1.0) ne permet pas au cycle du PREGEC de répondre de façon appropriée à l'évolution de la demande d'informations. L'utilisation d'une méthodologie uniforme qui capitalise sur les bonnes pratiques des parties prenantes du PREGEC et d'autres acteurs, conjuguée à un effort de leadership et de coordination, est essentielle pour garantir l'application de l'analyse sexospécifique qui sera recommandée dans le Cadre harmonisé actualisé. En outre, les parties prenantes du PREGEC et les autres acteurs devront déterminer dans quelle mesure cette initiative rivalise avec d'autres priorités et d'autres besoins en matière de ressources allouées au renforcement des SAP-SAN. 

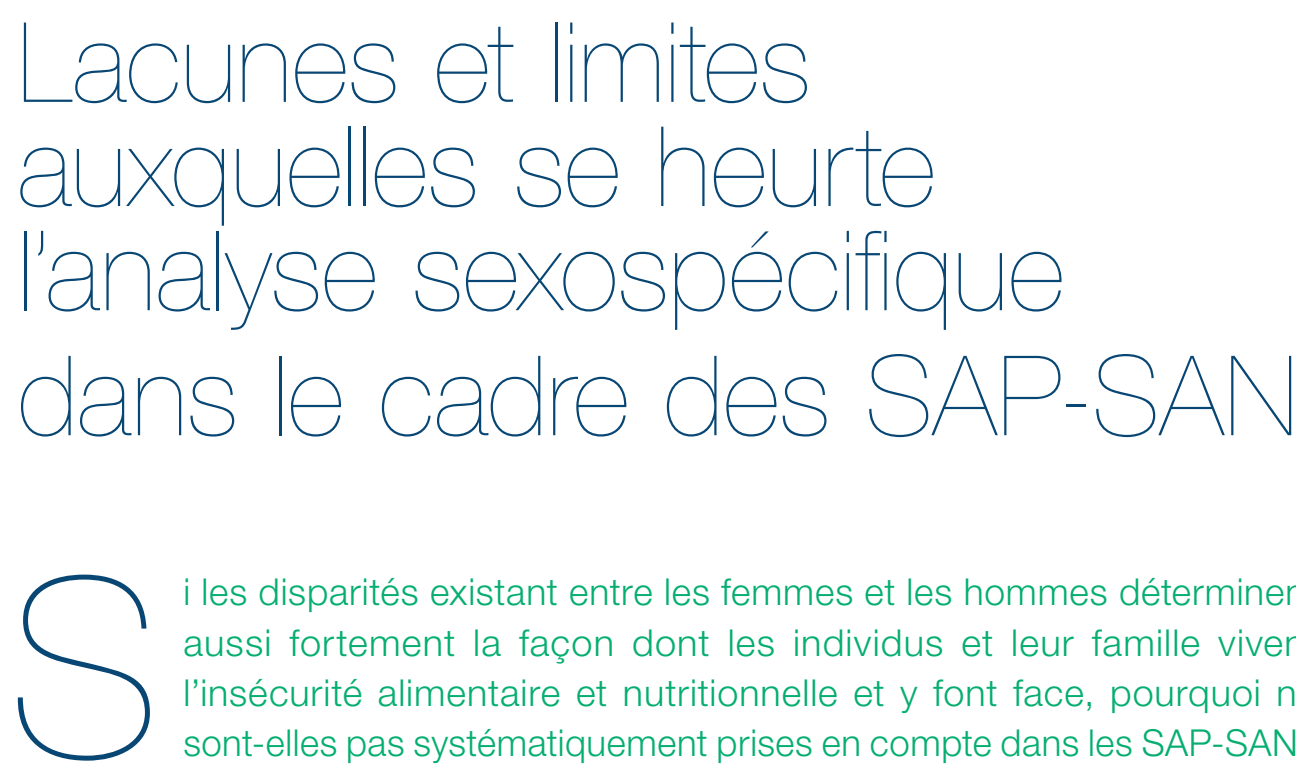

i les disparités existant entre les femmes et les hommes déterminent aussi fortement la façon dont les individus et leur famille vivent l'insécurité alimentaire et nutritionnelle et y font face, pourquoi ne sont-elles pas systématiquement prises en compte dans les SAP-SAN? Les outils et les mécanismes utilisés demeurent insuffisamment sensibles à ces disparités, alors que les faits montrent que s'ils l'étaient davantage, non seulement la situation des plus vulnérables s'améliorerait, mais les efforts de prévention et de gestion des crises seraient aussi plus efficaces. Cette situation résulte de plusieurs difficultés. Chez l'ensemble des parties prenantes du PREGEC, il manque, par exemple, des spécialistes de la problématique femmes-hommes, ainsi qu'une connaissance élémentaire des concepts que recouvre le principe de l'égalité entre les sexes. Oxfam a ainsi reconnu en 2017 que l'insensibilité de son SAP aux disparités entre femmes et hommes et le fait que ses données ne soient pas ventilées par sexe avaient eu pour effet d'amoindrir l'impact de ses interventions en Somalie, où les inégalités entre les sexes sont particulièrement fortes. Outre une méconnaissance du problème, il peut en résulter un manque d'intérêt pour celui-ci dans la mesure où l'importance déterminante que revêt l'analyse sexospécifique pour la sécurité alimentaire et nutritionnelle n'est pas pleinement reconnue.

Même si la mise en place de politiques sur l'égalité femmes-hommes constitue une première étape importante pour assurer que l'objectif d'égalité entre les sexes infuse dans l'ensemble des politiques, cela ne garantit pas que les SAP-SAN et leurs équipes prendront en compte les aspects sexospécifiques. Il ressort d'entretiens avec le CILSS, la FAO et le ministère de l'Agriculture des pays de la région que les politiques en faveur de l'égalité femmes-hommes ne sont pas diffusées et que, par conséquent, le personnel peut tout simplement en ignorer l'existence ${ }^{14}$. De même, lorsqu'il existe effectivement des directives solides sur l'utilisation de l'analyse sexospécifique dans les SAP-SAN, elles ne sont pas nécessairement adoptées et appliquées de façon généralisée, d’où l'absence, dans le rapport final, de recommandations basées sur ces aspects sexospécifiques. Par exemple, et comme il a été précédemment indiqué, la Stratégie conjointe du PAM et de la FAO sur les systèmes d'information 
souligne l'importance de l'analyse sexospécifique pour la surveillance de la sécurité alimentaire et nutritionnelle, et elle exige que soient prises en considération les disparités existant entre les sexes quant à la situation en matière de sécurité alimentaire. Or, très rares sont les rapports mondiaux de la FAO sur l'alerte et l'intervention précoces qui présentent des recommandations étayées par une analyse sexospécifique. Par conséquent, bien que cette Stratégie conjointe soit relativement sensible à la problématique femmes-hommes et contienne des directives solides en la matière, le SAP de la FAO ne tient pas pleinement compte des exigences qui découlent de ces directives.

L'insuffisance des ressources financières et humaines peut expliquer le manque de compétences en matière de problématique femmes-hommes dans beaucoup d'organisations y compris dans celles qui possèdent une politique en faveur de l'égalité entre les sexes, ainsi que des cadres et des outils d'aide au renforcement des capacités. Ceci conduit à penser qu'il n'existe pas de volonté d'investir dans ce domaine et qu'aucune impulsion n'est donnée dans ce sens. La version 1.0 du Cadre harmonisé en offre une bonne illustration : la problématique femmes-hommes y est évoquée exclusivement au sujet des facteurs nutritionnels liés aux nourrissons, aux jeunes enfants et aux femmes, avec une distinction entre les femmes non enceintes et les femmes enceintes et allaitantes (CILSS, 2016). Par conséquent, les parties prenantes du PREGEC ne parlent de la problématique femmes-hommes que lorsqu'il est question de nutrition. Les informations nutritionnelles concernant ces catégories de personnes sont essentielles, surtout dans le contexte des SAP-SAN. Toutefois, bien que la surveillance des besoins nutritionnels des femmes et des enfants soit importante, elle n'est pas suffisante pour étudier de façon suivie les aspects sexospécifiques de la sécurité alimentaire et nutritionnelle. Limiter l'analyse à ce seul aspect témoigne d'une connaissance technique très limitée des dimensions sexospécifiques de la sécurité alimentaire et nutritionnelle. En effet, l'analyse sexospécifique nécessite en principe la collecte de données sur les femmes, les filles, les hommes et les garçons en les séparant par groupes d'âge, afin d'évaluer les comportements et les pratiques propres à éclairer la planification des efforts de prévention et de gestion des crises.

Comme le PREGEC repose sur un ensemble d'informations et d'analyses provenant de sources diverses, il est fortement tributaire de la qualité et couverture des informations et sources. Une grande part de ces données provient d'organismes statistiques gouvernementaux et nationaux, ce qui génère des difficultés lorsque la majeure partie des données n'est pas accessible au public et donc impossible à analyser, et que celles qui sont disponibles ne sont pas toujours ventilées par sexe et par âge (PAM, 2016c). De même, pour certaines parties prenantes du PREGEC, la collecte d'informations sur les disparités entre les sexes est rendue plus difficile à cause de problèmes tels que le faible nombre de recenseurs ou les contraintes financières. De plus, lorsque des parties prenantes du PREGEC qui recueillent des DVSA 
présentent des indicateurs sexospécifiques, ces informations ne sont pas prises en compte dans le cycle du PREGEC ou dans le Cadre harmonisé pour combler les lacunes que comportent les SAP en la matière.

L'intégration de l'analyse sexospécifique dans le cycle du PREGEC peut aussi se heurter à des obstacles d'ordre méthodologique. Certaines parties prenantes ont déclaré craindre que, dans le cas de l'évaluation de la sécurité alimentaire et nutritionnelle fondée sur les zones géographiques et sur les prix (qui est prévue dans le Cadre harmonisé), les DVSA et l'analyse sexospécifique ne soient plus difficiles à utiliser que les données issues des enquêtes sur les ménages. La question se pose aussi avec les SAP qui s'appuient en grande partie sur l'observation des variations du climat et des changements saisonniers et font donc beaucoup appel aux données météorologiques. Par ailleurs, le manque de capacités techniques et de ressources freine considérablement l'amélioration des méthodes. Oxfam a souligné combien il avait été difficile pour l'Unité d'analyse de la sécurité alimentaire et de la nutrition et l'initiative IPC en Somalie, par exemple, de fournir des DVSA. En raison du grand nombre d'indicateurs pour lesquels des données sont recueillies et de la pression qui s'exerce pour que la collecte et la communication de ces données s'effectuent dans les temps, une ventilation plus fine de celles-ci, notamment par sexe et par âge, ne se ferait pas sans mal. Cela tient en grande partie à un manque de capacités, selon Oxfam, ainsi qu'à un manque de prise en compte des risques liés à la perpétuation d'une programmation qui méconnaît les disparités existant entre les sexes (Feeny, 2017).

Étant donné les liens étroits qui relient la problématique femmes-hommes et la sécurité alimentaire et nutritionnelle, il est important d'observer et d'analyser l'évolution de la situation des femmes et des filles. Les modalités actuelles d'incorporation des données provenant des SAP dans le PREGEC ne sont peut-être pas favorables à l'intégration de DVSA et d'indicateurs sexospécifiques (PAM, 2016c). Cependant, ces DVSA peuvent être apportées par d'autres canaux et/ou à travers la participation de spécialistes de la problématique femmes-hommes au processus du PREGEC. Des capacités seront sans doute nécessaires pour pouvoir recueillir les données et les ventiler entièrement, mais ne pas répondre à cette exigence risque d'empêcher la fourniture des informations requises et de compromettre ainsi la pertinence du PREGEC.

Les guides et les ressources liées à la problématique femmes-hommes soulignent l'importance de l'analyse sexospécifique, et proposent des bonnes pratiques à suivre pour la réaliser. Néanmoins, la mise en place de SAP sensibles aux disparités entre les sexes ne suscite pas autant d'intérêt. Cela vaut non seulement pour le secteur de la sécurité alimentaire et nutritionnelle, mais aussi pour tous les secteurs qui utilisent des SAP, comme le montre le tableau 4 sur les politiques des parties prenantes du PREGEC en faveur de l'égalité femmes-hommes qui font référence aux SAP-SAN. Ce problème a pour effet d'entamer la confiance avec laquelle les parties 
prenantes pourraient adopter et appliquer des mesures propres à renforcer l'utilisation de l'analyse sexospécifique dans le cadre des SAP et à permettre la standardisation de cet exercice.

Les lacunes et autres obstacles exposés ci-dessus traduisent une réalité évidente : l'absence d'action coordonnée là où un ensemble d'éléments de base est déjà en place. La plupart des parties prenantes du PREGEC reconnaissent l'importance de l'analyse sexospécifique et la majorité d'entre elles ont d'ores et déjà commencé à prendre des initiatives pour renforcer leurs propres capacités en la matière. Il apparaît aussi que les ressources d'appui, les compétences extérieures et les données primaires nécessaires existent déjà, ne serait-ce que de façon basique. Il est donc assez clair que le principal obstacle réside dans le fait que, le Cadre harmonisé ne comporte pas de directives détaillées sur l'intégration de l'analyse sexospécifique dans les SAP-SAN. Par conséquent, l'élaboration de la version 2.0 du Cadre harmonisé est une occasion propice pour définir un cadre d'analyse, des outils et des activités de formation sur la problématique femmes-hommes pour le PREGEC.

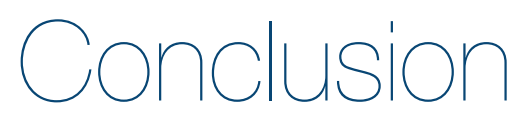

Cette note a discuté l'importance de prendre en compte les aspects sexospécifiques de la sécurité alimentaire et nutritionnelle dans les SAP-SAN. Elle a présenté une vue d'ensemble des initiatives mises en place par les membres du PREGEC pour mieux capturer l'influence des disparités hommes-femmes dans leurs systèmes d'alerte. Il apparaît que les effets positifs d'une prise en compte des effets liés au genre dans les SAP-SAN sont documentés dans un corpus toujours plus grand de recherches. L'expérience des membres du PREGEC dans d'autres domaines d'action publique souligne également ces effets positifs. Tout paraît donc en place pour intégrer les aspects de la sécurité alimentaire liés au genre dans les SAP-SAN.

Intégrer les problématiques femmes-hommes dans les SAP-SAN n'est cependant pas chose facile. Le manque de compétences et de budget, ou encore les pressions présentes dans les contextes fragiles et les situations d'urgence, sont des freins importants. C'est pourquoi les parties prenantes du PREGEC devront faire preuve d'une volonté sans faille sur la question des rapports entre genre et insécurité alimentaire. Concrètement, cela implique d'y consacrer des moyens financiers et de travailler à l'harmonisation et à la diffusion des politiques existantes sur l'égalité femmes-hommes. Le PREGEC comporte toujours des lacunes, mais il est indéniable que les membres font déjà des efforts pour améliorer les SAP et le Cadre harmonisé. Il faut donc poursuivre dans cette voie tout en insistant toujours tant et plus sur 
l'importance de faire un suivi précis et durable des dimensions sexospécifiques de la sécurité alimentaire.

L'intégration de la problématique femmes-hommes dans les SAP-SAN est indispensable pour assurer la sécurité alimentaire et nutritionnelle et, d'une manière plus générale, l'avènement de l'égalité entre les sexes. Par conséquent, toutes les parties prenantes du PREGEC ont intérêt à considérer la problématique femmes-hommes comme une dimension essentielle à prendre en compte lors de l'élaboration des SAP-SAN, depuis la collecte de données ventilées par sexe et par âge jusqu'à l'étude de l'influence des dynamiques socioculturelles. Une plus grande participation des femmes à toutes les étapes de la planification et de la mise en œuvre des SAP-SAN permettra d'améliorer la surveillance et la prévention. Rendre les SAP-SAN sensibles aux spécificités de genre est donc un objectif de développement en tant que tel.

Le Cadre harmonisé a été créé dans l'esprit de dépasser les limites que comportaient à l'époque les outils sur lesquels reposaient les SAP-SAN. Le travail et la coopération sur les SAP-SAN doit être envisagé comme un processus d'amélioration continue. La présente note s'inscrit dans cette optique. Alors que la mise à jour du Cadre harmonisé approche, elle vise à pointer du doigt les limites de la version 1.0 du Cadre harmonisé, et à présenter les possibilités qui s'offrent pour mieux intégrer la problématique femmes-hommes dans la version 2.0 et améliorer ainsi l'efficience globale des SAP-SAN.

\section{Notes}

$1 \quad$ Le Cadre harmonisé donne aux pays les moyens d'évaluer l'insécurité alimentaire et nutritionnelle sur une base consensuelle. II permet aux gouvernements et aux acteurs humanitaires de la région de prendre des décisions en connaissance de cause pour prévenir, atténuer, anticiper et gérer les crises alimentaires.

2 Le RCPA rassemble des pays du Sahel et de l'Afrique de l'Ouest, des organismes de coopération bilatérale et multilatérale, des organismes humanitaires et des ONG internationales, des organisations professionnelles agricoles, des organisations de la société civile et du secteur privé, ainsi que des systèmes d'information internationaux et régionaux, autour de la CEDEAO, de I'UEMOA et du CILSS. Voir OCDE, «RCPA - Réseau de prévention des crises alimentaires - $\mathrm{A}$ propos du Réseau », www.oecd.org/site/rpca/aboutus.

Liste non exhaustive.

IPC Analysis Portal. IPC \& Cadre Harmonise in West Africa and the Sahel.

www.ipcinfo.org/ipcinfo-website/where-what/cadre-harmonise-in-west-africa-and-the-sahel/en. Liste non exhaustive.

Entretiens avec des parties prenantes du PREGEC.

Entretiens avec le CILSS et FEWS NET.

Entretien avec la FAO.

Burundi, Djibouti, Éthiopie, Kenya, Rwanda, Somalie, Soudan du Sud et Ouganda.

PAM (2016), "VAM Gender Analysis: Resource package", VAM Resource Center - Gender, World Food

Programme (launched in 2016); PAM (2017), "Boîte à outils relative à la problématique hommes-

femmes”, (document évolutif, mise au point en cours). 
12 vam.wfp.org

13 Entretiens avec des spécialistes de la problématique femmes-hommes du PAM et du CILSS travaillant sur la deuxième version du manuel du Cadre harmonisé.

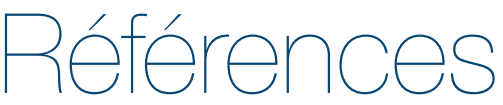

ACF (2014), " Politique ACF en matière de gendre : Accroître l'impact de l'action d'ACF en développant des programmes sensibles à l'égalité des sexes ", Action Contre la Faim International, www.actionagainsthunger.org/sites/default/files/publications/ACF_Gender_ Policy_2014_FR_1.pdf.

Allen, T., P. Heinrigs et I. Heo, " Agriculture, alimentation et emploi en Afrique de l'Ouest ", Notes ouest-africaines, n 14, Éditions OCDE, Paris, 2018, https://doi.org/10.1787/56d463a9-fr.

Banque mondiale (s.d.), « Gender Data Portal | Country - Sub-Saharan Africa », http://datatopics. worldbank.org/gender/country/sub-saharan-africa (consulté le 1 octobre 2018).

BRIDGE, (2014), Genre et sécurité alimentaire - Vers une sécurité alimentaire et nutritionnelle équitable en termes de genre, Bridge - Institute of Development Studies, Brighton.

CARE (2017), "Food security, nutrition, climate change resilience and gender. Policies for smallscale farmers, Tanzania ", www.careevaluations.org/wp-content/uploads/Policies-for-smallscale-farmers.pdf.

CEA (2014), "An Assessment of Gender Mainstreaming into Food Security Initiatives of West African Intergovernmental Organisations: Study on Gender Mainstreaming " Commission économique pour l'Afrique des Nations unies, www.uneca.org/sites/default/files/ PublicationFiles/gendermainstream_mep2_ok_jan26-2015.pdf.

CILSS (2016), Manuel du Cadre harmonisé Version 1.0 - Cadre harmonisé d'identification et d'analyse des zones à risque et des populations en insécurité alimentaire et nutritionnelle au Sahel et en Afrique de l'Ouest, Comité permanent interखÉtats de lutte contre la sécheresse dans le Sahel, https://reliefweb.int/sites/reliefweb.int/files/resources/ch_manual_en_003.pdf.

Demetriades, J. and E. Esplen (2008), "The Gender Dimensions of Poverty and Climate Change Adaptation ", IDS Bulletin 39, n 4, pp. 24 31, https://doi.org/10.1111/j.1759-5436.2008. tb00473.x.

DHS Program (s.d.), "The DHS Program - Quality information to plan, monitor and improve population, health, and nutrition programs ", www.dhsprogram.com/ (consulté le 2 octobre 2018).

El Rhomri, I. (2015), "Vers l'intégration de l'approche Genre Dans La Prévention et La Gestion de l'insécurité Alimentaire : Analyse Critique Du Cadre d'analyse HEA/AEM », Rapports de recherche Oxfam, www.researchgate.net/publication/317604849_Vers_l'integration_ de_l'approche_genre_dans_la_prevention_et_la_gestion_de_l'insecurite_alimentaire_ Analyse_critique_du_cadre_d'analyse_HEAAEM.

FAO (s.d.-a), "Dimitra: Dimitra Clubs », www.fao.org/dimitra/dimitra-clubs/en, Organisation des Nations unies pour l'alimentation et l'agriculture, (consulté le 19 septembre 2018).

FAO (s.d.-b), "Early Warning Early Action: FAO in Emergencies », Organisation des Nations unies pour l'alimentation et l'agriculture, www.fao.org/emergencies/fao-in-action/ewea/en.

FAO (s.d.-c), " Gender and Land Rights Database », Organisation des Nations unies pour l'alimentation et l'agriculture, http://www.fao.org/gender-landrights-database/en.

FAO (s.d.-d), "GIEWS - Global Information and Early Warning System », Organisation des Nations unies pour l'alimentation et l'agriculture, www.fao.org/giews/en, (consulté le 19 septembre 2018).

FAO (s.d.-e), "Sécurité alimentaire et résilience aux changements climatiques au Sahel et en Afrique de l'Ouest : FAO in Emergencies ", Organisation des Nations unies pour l'alimentation et l'agriculture, www.fao.org/emergencies/fao-in-action/projects/detail/en/c/1150161 (consulté le 19 septembre 2018).

FAO (2017a), "Final evaluation of the project: Food Security and Nutrition Analysis Unit for Somalia ", Organisation des Nations unies pour l'alimentation et l'agriculture, http://www.fao. org/3/a-bd688e.pdf. 
FAO (2017b), "Global Early Warning - Early Action report on Food Security and Agriculture ", Organisation des Nations unies pour l'alimentation et l'agriculture, http://www.fao.org/3/ai6790e.pdf

FAO (2017c), « Guidelines for the assessment of gender mainstreaming », Organisation des Nations unies pour l'alimentation et l'agriculture, http://www.fao.org/3/a-bd714e.pdf.

FAO (2017d), "L'approche Cash+ au Sahel : un outil pour le renforcement de la résilience ", Organisation des Nations unies pour l'alimentation et l'agriculture, http://www.fao.org/3/ai7412f.pdf.

FAO (2016a), " Gender, Food Security and Nutrition in Protracted Crises ", Organisation des Nations unies pour l'alimentation et l'agriculture, www.fao.org/3/a-i6630e.pdf, (consulté le 19 septembre 2018).

FAO (2016b), " Gender-Responsive Disaster Risk Reduction in the Agriculture Sector ", Organisation des Nations unies pour l'alimentation et l'agriculture, www.fao.org/3/b-i6096e. pdf

FAO (2015), « Incorporating Gender Concerns in the Resilience Agenda Experiences from FAO ", Organisation des Nations unies pour l'alimentation et l'agriculture, https://www. climateinvestmentfunds.org/sites/cif_enc/files/FAO_Gender_in_Resilience.pdf

FAO et PAM (2008), "SEAGA (Socio-Economic and Gender Analysis) Emergency and Rehabilitation Programmes ", http://www.fao.org/tempref/docrep/fao/008/y5702e/y5702e.pdf.

FAO, Banque mondiale et FIDA (2015), "Gender in Climate Smart Agriculture: Module 18 for the Gender in Agriculture ", http://www.fao.org/3/a-i5546e.pdf.

Feeny, E. (2017), « From Early Warning to Early Action in Somalia: What Can We Learn to Support Early Action to Mitigate Humanitarian Crises? ", Oxfam, https://doi.org/10.21201/2017.0834.

FEWSNET (2017), "West Africa - Enhanced Market Analysis ", https://reliefweb.int/sites/reliefweb. int/files/resources/FEWS\%20NET\%20West\%20EMA_Final_20171201.pdf.

GBVIMS (s.d.), " GBVIMS: Gender-Based Violence Information Management System ”, www. gbvims.com (consulté le 2 octobre 2018).

Gnisci, D. (2016), "Women's Roles in the West African Food System: Implications and Prospects for Food Security and Resilience », West African Papers, $n^{\circ}$ 3, OECD Publishing, Paris, https:// doi.org/10.1787/5j|p|4mh1hxn-en.

Hendriks, S.L. et E. Maunder (2006), «Identification of Food Security Indicators for FIVIMS.ZA ", Research Gate, www.researchgate.net/publication/272019422_Identification_of_food_ security_indicators_for_FIVIMSZA.

CPI (2017), "Égalité des genres et secteurs spécifiques : Sécurité alimentaire », In Le guide des genres pour les actions humanitaires, Comité Permanent Interorganisations, https://reliefweb. int/sites/reliefweb.int/files/resources/iasc_gender_handbook_2017.pdf.

IFPRI (2012), "Women's Empowerment in Agriculture Index (WEAl) Booklet », Institut international de recherche sur les politiques alimentaires, www. ifpri.org/publication/womensempowerment-agriculture-index.

Le Masson, V. et al. (2015), "Gender and Resilience Working Paper », ODI, aout, www.odi.org/ sites/odi.org.uk/files/odi-assets/publications-opinion-files/9890.pdf.

mVAM. The Blog (2015), "Ladies - We Can't Hear y'all! Addressing Gender Issues in Mobile Data Collection in West Africa ", MVAM: THE BLOG, 24 novembre, http://mvam.org/2015/11/24/ ladies-we-cant-hear-yall-addressing-gender-issues-in-mobile-data-collection-in-west-africa/.

OCDE (s.d.), « Gender Data - OECD », www.oecd.org/gender/data (consulté le 2 octobre 2018).

OCDE (s.d.), «RCPA - The Food Crisis Prevention Network - About us ", www.oecd.org/site/rpca/ aboutus (consulté le 9 octobre 2018).

OMS (s.d.), «WHO | Global Health Observatory (GHO) data », Organisation mondiale de la santé, http://www.who.int/gho/en/ (consulté le 1 octobre 2018).

ONU Femmes (2012), "Gender-Responsive Early Warning: Overview and How-to Guide ", www. unwomen.org/ /media/Headquarters/Media/Publications/en/04EGenderResponsiveEarlyWar ning.pdf.

OSCE/ODIHR (2009), "Gender and Early Warning Systems: An Introduction », www.osce.org/ odihr/40269?download=true.

Oxfam (2013), "Oxfam Minimum Standards for Gender in Emergencies », https://d1tn3vj7xz9fdh. cloudfront.net/s3fs-public/file_attachments/ml-oxfam-gender-emergencies-minimumstandards-291113-en_0_0.pdf.

PAM (s.d.-a), " Dataviz - World Food Programme ", Programme alimentaire mondial, http://dataviz. vam.wfp.org (accessed 18 September 2018). 
PAM (s.d.-b), « Nigeria - mVAM Monitoring ", Programme alimentaire mondial, www.wfp.org/ content/nigeria-mvam-monitoring, (accessed 20 September 2018).

PAM (s.d.-c), « West Africa Gender and Market Initiative: Case studies », VAM Resource Center, World Food Programme, Dakar, www.wfp.org/content/gender-and-markets-initiativewest-and-central-africa-country-case-studies?_ga=2.75610647.363570283.1553580828341602115.1553462992 (accessed 18 September 2018).

PAM (s.d.-d), "West Africa Gender and Market Initiative: Resource Centre ", VAM Resource Center, Programme alimentaire mondial, Dakar, https://resources.vam.wfp.org/node/105, (accessed 18 September 2018).

PAM (s.d.-e), "West Africa Gender and Markets Initiative: Working towards Zero Hunger by Empowering West African Women ", VAM Resource Center, Programme alimentaire mondial, Dakar, https://resources.vam.wfp.org/node/106.

PAM (s.d.-f), " WFP VAM Home Page », http://vam.wfp.org (accessed 18 September 2018).

PAM (2018), « mVAM Bulletin \#10 Nigeria August 2018 », Programme alimentaire mondial, http:// dataviz.vam.wfp.org/nigeria-mvam-bulletin-10-aug-2018 (accessed 18 September 2018).

PAM (2017), " Can social network analysis inform African development policies ? An application to food security, market analysis and gender ", Programme alimentaire mondial, https://docs.wfp.org/api/documents/WFP-0000021022/download/?_ ga $=2.187476904 .1284600164 .1538317682-621711467.1535536870$.

PAM (2016a), " Description of an Adapted Gender Marker System for Assessments ", Programme alimentaire mondial, https://documents.wfp.org/stellent/groups/public/documents/ena/ wfp287835.pdf.

PAM (2016b), " Gender and Food Security Analysis: Guidance Document ", Programme alimentaire mondial, https://docs.wfp.org/api/documents/WFP-0000019670/download/? ga $=2.267315342 .1284600164 .1538317682-621711467.1535536870$.

PAM (2016c), "Gender and Markets in West Africa: Secondary Data Review ", Programme alimentaire mondial, Dakar, https://reliefweb.int/sites/reliefweb.int/files/resources/1518109173. Secondary\%20Data_en_v2.0.pdf.

PAM (2016d), « Guidance Note: Integrating gender into vulnerability and food security analysis ", Programme alimentaire mondial, Dakar, https://resources.vam.wfp.org/sites/default/files/ VAM\%20Gender\%20Analysis\%20Guidance\%20Note.pdf.

PAM (2016e), " Technical Note on use of gender- and empowerment-integrated market assessment surveys ", Programme alimentaire mondial, Dakar, https://resources.vam.wfp.org/ sites/default/files/Technical\%20Note\%20English_Updated\%2021\%20April.pdf.

PAM (2015), " Consolidated Approach to Reporting Indicators of Food Security (CARI) Guidelines ", Programme alimentaire mondial, www.wfp.org/content/consolidated-approach-reportingindicators-food-security-cari-guidelines.

RPCA (2018), "Concertation technique du DISPOSITIF RÉGIONAL DE PRÉVENTION ET DE GESTION DES CRISES ALIMENTAIRES (PREGEC) ", Dakar, www.cilss.int/index.php/le-cyclepregec-prevention-et-gestion-des-crises-alimentaires-au-sahel-et-en-afrique-de-louest/.

Save the Children (s.d.), "Gender Equality Resource Center », Resource Centre, https:// resourcecentre.savethechildren.net/our-thematic-areas/cross-thematic-areas/gender-equality (consulté le 19 septembre 2018).

Save the Children (2016), « Unequal Portions: Ending Malnutrition for every last child », https:// resourcecentre.savethechildren.net/library/unequal-portions-ending-malnutrition-every-lastchild.

Save the Children (2014), "Save the Children Gender Equality Program Guidance and Toolkit: Engendering Transformational Change ", https://resourcecentre.savethechildren.net/ node/8503/pdf/gender_equality_program_toolkit_2014.pdf.

Schmeidl, S. (2001), "Gender and Early Warning », In Hommes Armés, Femmes Aguerries, pp. 51 61. Graduate Institute Publications, https://doi.org/10.4000/books.iheid.6137.

UNESCO/UIS (s.d.), " Gender Equality in Education », http://uis.unesco.org/en/topic/genderequality-education (consulté le 2 octobre 2018).

UNISDR, PNUD et IUCN (2009), «Intégration de la dimension de genre dans la réduction des risques de catastrophes ", Geneva, www.unisdr.org/files/9922 publicationintgrationdugenredanslar.pdf.

UNISDR (2015), « Sendai Framework for Disaster Risk Reduction 2015 - 2030 » United Nations Office for Disaster Risk Reduction, www.unisdr.org/we/inform/publications/43291.

UNSD et ONU Femmes (n.d.), " Evidence and Data for Gender Equality (EDGE) project », https:// unstats.un.org/edge/.

USAID (2017), "Food security desk review for Niger », USAID Office of Food for Peace, pp. 127. 


\section{Annex I : Problématique femmes-hommes et marchés en Afrique de l'Ouest: liste des sources de données secondaires et description succincte août 2016 (PAM)}

\begin{tabular}{|c|c|c|c|}
\hline Nom & Source/ Org. & Type & Description \\
\hline Genre et éducation & $\begin{array}{l}\text { Institut de } \\
\text { statistique de } \\
\text { I'UNESCO }\end{array}$ & Recueil d'indicateurs & $\begin{array}{l}\text { Indicateurs clés, ventilés par sexe et par âge, servant à } \\
\text { suivre l'évolution des disparités entre les sexes dans le } \\
\text { domaine de l'éducation au niveau mondial. }\end{array}$ \\
\hline $\begin{array}{l}\text { Base de données } \\
\text { Genre et le droit à la } \\
\text { terre }\end{array}$ & FAO & Base de données & $\begin{array}{l}\text { La base de données Genre et le droit à la terre a été mise } \\
\text { en place par la FAO en 2010, afin de mettre en lumière les } \\
\text { principaux facteurs d'ordre politique, juridique et culturel } \\
\text { qui influent sur l'exercice, par les femmes, de leurs droits } \\
\text { fonciers dans le monde entier. Elle sert aussi de plateforme } \\
\text { pour aborder et traiter les questions concernant les dispari- } \\
\text { tés entre les sexes et la terre et apporter des informations } \\
\text { sur ce sujet, en prenant appui sur } 84 \text { profils-pays, des } \\
\text { statistiques sur le régime foncier ventilées par sexe, ainsi } \\
\text { que l'Outil d'évaluation de la législation pour un régime } \\
\text { foncier équitable entre les sexes (LAT). }\end{array}$ \\
\hline $\begin{array}{l}\text { Statistiques Genre et } \\
\text { parité hommes- } \\
\text { femmes }\end{array}$ & $\begin{array}{l}\text { Banque } \\
\text { mondiale }\end{array}$ & Base de données & $\begin{array}{l}\text { La base de données statistiques Genre et parité hommes- } \\
\text { femmes contient des indicateurs sur des thèmes essentiels } \\
\text { de la problématique femmes-hommes, dont la démogra- } \\
\text { phie, l'éducation, la santé, la population active et la } \\
\text { participation à la vie politique. S'y trouvent également des } \\
\text { indicateurs sur certains aspects de l'autonomisation, dont } \\
\text { le pouvoir de décision en matière de santé, les activités } \\
\text { économiques et la dynamique interne aux ménages. II } \\
\text { s'agit du corpus de ressources le plus détaillé et le plus } \\
\text { complet du monde sur la dynamique de la problématique } \\
\text { femmes-hommes, notamment en ce qui concerne les } \\
\text { données ventilées par sexe et par âge. }\end{array}$ \\
\hline $\begin{array}{l}\text { Statistiques sur la } \\
\text { problématique } \\
\text { femmes-hommes } \\
\text { (EDGE) }\end{array}$ & $\begin{array}{l}\text { Division de la } \\
\text { statistique des } \\
\text { Nations Unies }\end{array}$ & Recueil d'indicateurs & $\begin{array}{l}52 \text { indicateurs quantitatifs et } 11 \text { indicateurs qualitatifs } \\
\text { couvrant les normes et les lois nationales qui se rapportent } \\
\text { à la question de l'égalité femmes-hommes. }\end{array}$ \\
\hline
\end{tabular}




\begin{tabular}{|c|c|c|c|}
\hline $\begin{array}{l}\text { Base de données } \\
\text { Égalité femmes- } \\
\text { hommes, institutions } \\
\text { et développement } \\
\text { (EID) }\end{array}$ & OCDE & Base de données & $\begin{array}{l}\text { La base de données EID est destinée à aider les cher- } \\
\text { cheurs et les responsables de l'élaboration des politiques à } \\
\text { déterminer et analyser les obstacles qui entravent la } \\
\text { progression des femmes dans la vie économique. Elle } \\
\text { couvre un total de } 160 \text { pays et contient } 60 \text { indicateurs sur } \\
\text { les discriminations sexistes. Elle a été élaborée à partir de } \\
\text { sources diverses et regroupe de façon systématique et } \\
\text { cohérente les données factuelles existant à l'heure actuelle } \\
\text { sur la situation socioéconomique des femmes. } \\
\text { Caractéristique particulièrement intéressante, cette base } \\
\text { de données comporte des variables institutionnelles qui } \\
\text { vont du comportement à l'intérieur du ménage jusqu'aux } \\
\text { normes sociales. Les informations concernant les pratiques } \\
\text { culturelles et coutumières qui influent sur la progression } \\
\text { des femmes dans la vie économique sont codées de façon } \\
\text { à permettre la mesure du niveau de discrimination. }\end{array}$ \\
\hline $\begin{array}{l}\text { Système de gestion } \\
\text { des informations sur } \\
\text { la violence basée sur } \\
\text { le genre }\end{array}$ & $\begin{array}{l}\text { FNUAP/ IRC/ } \\
\text { HCR/ UNICEF }\end{array}$ & $\begin{array}{l}\text { Système de gestion de } \\
\text { l'information }\end{array}$ & $\begin{array}{l}\text { Le Système de gestion des informations sur la violence } \\
\text { basée sur le genre est un dispositif multidimensionnel qui } \\
\text { permet aux acteurs humanitaires intervenant face à des } \\
\text { actes de violence sexiste de procéder avec efficacité et en } \\
\text { toute sécurité à la collecte, au stockage, à l'analyse et à la } \\
\text { communication des données dégagées des déclarations } \\
\text { des survivantes de la violence sexiste. }\end{array}$ \\
\hline $\begin{array}{l}\text { Global Health } \\
\text { Observatory Data } \\
\text { Repository (GHO) }\end{array}$ & OMS & $\begin{array}{l}\text { Données sur la santé, } \\
\text { ventilées par sexe et } \\
\text { par âge }\end{array}$ & $\begin{array}{l}\text { Ce répertoire de données de l'Observatoire mondial de la } \\
\text { santé permet d'accéder à plus de } 1000 \text { indicateurs sur } \\
\text { des questions prioritaires concernant la santé, dont la } \\
\text { mortalité et la charge de morbidité, les Objectifs du } \\
\text { millénaire pour le développement (la nutrition chez les } \\
\text { enfants, la santé des enfants, la santé maternelle et } \\
\text { procréative, la vaccination, le VIH/sida, la tuberculose, le } \\
\text { paludisme, les maladies négligées, l'eau et } \\
\text { l'assainissement), les maladies non transmissibles et les } \\
\text { facteurs de risque, les maladies à propension épidémique, } \\
\text { les systèmes de santé, I'hygiène de l'environnement, la } \\
\text { violence et les blessures, ainsi que l'équité, entre autres. } \\
\text { En outre, I'Observatoire mondial de la santé permet } \\
\text { d'accéder en ligne à la synthèse annuelle des données sur } \\
\text { la santé que l'OMS effectue pour ses États membres, les } \\
\text { Statistiques mondiales de la santé. }\end{array}$ \\
\hline $\begin{array}{l}\text { Ensembles de } \\
\text { données de l'IFPRI }\end{array}$ & IFPRI & $\begin{array}{l}\text { Ensembles de } \\
\text { données }\end{array}$ & $\begin{array}{l}\text { II s'agit d'un corpus de différents ensembles de données } \\
\text { comprenant des données ventilées par sexe et par âge et } \\
\text { des statistiques sexospécifiques pour un éventail de pays } \\
\text { et de régions. }\end{array}$ \\
\hline
\end{tabular}




\begin{tabular}{|c|c|c|c|}
\hline $\begin{array}{l}\text { Indicateurs clés du } \\
\text { marché du travail } \\
\text { (KILM) }\end{array}$ & BIT & Système d'information & $\begin{array}{l}\text { Les indicateurs clés du marché du travail (KILM) constitu- } \\
\text { ent le principal instrument d'information du Bureau } \\
\text { international du travail (BIT) sur le marché du travail. Les } \\
\text { premiers KILM ont été publiés en 1999. Ils sont devenus } \\
\text { depuis un produit phare du BIT et sont couramment utilisés } \\
\text { par les chercheurs et les décideurs du monde entier. La } \\
\text { plupart des données concernant l'activité sont ventilées par } \\
\text { sexe et, dans certains cas, par âge. }\end{array}$ \\
\hline $\begin{array}{l}\text { U.S. Census Interna- } \\
\text { tional Data Base }\end{array}$ & $\begin{array}{l}\text { United States } \\
\text { Census Bureau }\end{array}$ & Base de données & $\begin{array}{l}\text { Cette base de données contient des informations sur la } \\
\text { population, ventilées par sexe et par âge pour la plupart } \\
\text { des pays. Elle peut être utilisée lorsque les statistiques } \\
\text { nationales ne se prêtent pas à une ventilation par sexe et } \\
\text { par âge. }\end{array}$ \\
\hline SEGREGAT & BIT & Base de données & $\begin{array}{l}\text { Il s'agit d'une base de données sur l'emploi par sexe et par } \\
\text { catégorie professionnelle fine qui contient des statistiques } \\
\text { sur plus de } 80 \text { pays développés et en développement et } \\
\text { pour des années proches de 1970, 1980, } 1990 \text { et } 2000 \text {. } \\
\text { Ces statistiques ne sont pas toujours comparables d'un } \\
\text { pays à l'autre ou d'un point dans le temps à un autre, en } \\
\text { raison de différences concernant les sources dont } \\
\text { proviennent les données recueillies, la population de } \\
\text { travailleurs couverte et les classifications nationales. II est } \\
\text { toutefois possible de comparer des catégories profession- } \\
\text { nelles bien définies, comme celles des enseignants, des } \\
\text { médecins et des chauffeurs de taxi. }\end{array}$ \\
\hline $\begin{array}{l}\text { Base de données } \\
\text { statistiques de la } \\
\text { CEE-ONU }\end{array}$ & CEE-ONU & Base de données & $\begin{array}{l}\text { Cette base de données comporte une section sur la } \\
\text { problématique femmes-hommes et la population, subdivi- } \\
\text { sée en plusieurs sous-sections sur des thèmes tels que le } \\
\text { travail et l'économie, l'équilibre entre vie personnelle et vie } \\
\text { active, les migrations et la prise de décision. Elle présente } \\
\text { en outre des vues d'ensemble par pays. }\end{array}$ \\
\hline FAOSTAT & FAO & & $\begin{array}{l}\text { Elle contient des statistiques générales sur l'agriculture et } \\
\text { la sécurité alimentaires établies par la FAO. }\end{array}$ \\
\hline $\begin{array}{l}\text { Agri-Gender Statis- } \\
\text { tics }\end{array}$ & FAO & Base de données & $\begin{array}{l}\text { Cette base de données contient des instructions expliquant } \\
\text { comment obtenir des données et comment bien les } \\
\text { analyser en se plaçant dans une optique sexospécifique. } \\
\text { Les exemples que contient cette base de données } \\
\text { proviennent de recensements agricoles effectués dans les } \\
\text { pays suivants : Bénin (à paraître), Botswana (1993), } \\
\text { Burkina Faso (1993), Côte d'Ivoire (2000/2001), Éthiopie } \\
\text { (2001/2002), Gambie (2001), Guinée (2000/2001), Mali } \\
\text { (1999/2000), Mauritanie (2003/2004), Niger (2004 } \\
\text { - 2006), Sénégal (1998/1999), Tanzanie (2002/2003), } \\
\text { Togo (1996), Tunisie (1995) et Ouganda (2003). }\end{array}$ \\
\hline
\end{tabular}




\begin{tabular}{|c|c|c|c|}
\hline $\begin{array}{l}\text { Sahel Online Report- } \\
\text { ing System (ORS) }\end{array}$ & $\mathrm{BCAH}$ & Base de données & $\begin{array}{l}\text { L'ORS est un outil de suivi des performances qui permet } \\
\text { aux partenaires humanitaires participant aux processus de } \\
\text { planification interorganisations de rendre compte directe- } \\
\text { ment des réalisations issues des activités prévues dans le } \\
\text { Plan de réponse stratégique/Plan de réponse humanitaire. } \\
\text { Cette base de données a été conçue pour faciliter } \\
\text { l'échange d'informations et le suivi des résultats des } \\
\text { interventions humanitaires. } \\
\text { La base de données contient, pour la majorité des pays de } \\
\text { l'Afrique de l'Ouest, des informations sur la sécurité } \\
\text { alimentaire (nombre de personnes en situation d'insécurité } \\
\text { et la nutrition (chez les enfants, filles et garçons, ou chez } \\
\text { les femmes enceintes et allaitantes). Elle présente aussi } \\
\text { des chiffres sur la population, ventilés par sexe et par âge. }\end{array}$ \\
\hline $\begin{array}{l}\text { Les femmes dans le } \\
\text { monde } 2015\end{array}$ & $\begin{array}{l}\text { Statistiques des } \\
\text { Nations Unies }\end{array}$ & Base de données & $\begin{array}{l}\text { Le rapport Les femmes dans le monde } 2015 \text { contient huit } \\
\text { chapitres qui traitent de thèmes de préoccupation } \\
\text { essentiels des pouvoirs publics : la population et la famille, } \\
\text { la santé, l'éducation, le travail, le pouvoir et la prise de } \\
\text { décision, la violence contre les femmes, l'environnement et } \\
\text { la pauvreté. Pour chaque thème est appliquée une } \\
\text { approche fondée sur le cycle de vie, afin de mettre en } \\
\text { évidence le vécu des femmes et des hommes aux } \\
\text { différentes périodes de leur vie, depuis l'enfance et les } \\
\text { années formatrices, jusqu'à la vieillesse, en passant par la } \\
\text { vie professionnelle et les années de procréation. } \\
\text { Les statistiques et les analyses présentées sont fondées } \\
\text { sur l'examen approfondi et rigoureux d'un vaste ensemble } \\
\text { de données provenant d'organismes statistiques internatio- } \\
\text { naux et nationaux. Chaque chapitre fait le point sur les } \\
\text { lacunes que comportent les statistiques sexospécifiques, } \\
\text { en mettant en lumière les progrès réalisés en matière de } \\
\text { statistiques disponibles, les dernières avancées sur le plan } \\
\text { méthodologique, ainsi que les domaines qui exigent } \\
\text { davantage d'attention de la part de la communauté interna- } \\
\text { tionale. Parmi les chapitres les plus importants figurent } \\
\text { ceux qui concernent le travail et la pauvreté. Outre les } \\
\text { statistique. }\end{array}$ \\
\hline
\end{tabular}




\section{Annex II : Liste des organismes consultés}

\begin{tabular}{llll} 
Organisation & Contactée (Oui/Non) & Consultée (Oui/Non) \\
\hline Partenaires techniques & & \\
\hline ACF & OUI & OUI - deux agents \\
\hline CILSS & OUI & OUI - deux agents \\
\hline FAO & OUI & OUI - deux agents \\
\hline FEWSNET & OUI & OUI - trois agents \\
\hline OXFAM & OUI & NON \\
\hline Save the Children & OUI & NON \\
\hline UNICEF & OUI & OUI - deux agents \\
\hline PAM & OUI &
\end{tabular}

Partenaires financiers

\begin{tabular}{ll:l}
\hline UE/ECHO & OUI & OUI - un agent \\
\hline USAID & OUI & NON \\
\hline Direction politique & & NON \\
\hline ECOWAS & OUI & OUI - un agent
\end{tabular}





\section{Notes ouest-africaines}

\section{Intégrer l'analyse sexospécifique dans les systèmes d'alerte précoce pour la sécurité alimentaire et nutritionnelle en Afrique de l'Ouest}

L'intégration de la problématique femmes-hommes dans les systèmes d'alerte précoce est essentielle afin de prévenir et gérer les crises de façon plus équitable. Cette Note examine la prise en compte dans les systèmes d'alerte précoce pour la sécurité alimentaire et nutritionnelle (SAP-SAN) au Sahel et en Afrique de l'Ouest du genre. Elle identifie les lacunes aux niveaux national et régional. II en ressort que les partenaires et parties prenantes ont renforcé la capacité d'analyse sexospécifique de ces systèmes. Mais ces avancées n'ont pas abouti à une refonte uniforme des mécanismes conjoints auxquels font appel les SAP-SAN afin de les rendre plus sensibles aux disparités qui existent entre les sexes. Des efforts plus concertés s'imposent pour déterminer les aspects de la problématique femmes-hommes à intégrer dans les SAP-SAN et en assurer le suivi pour des systèmes de prévention et de gestion de crise plus équitables. Ce document présente des pistes pour appuyer ces efforts. 